\title{
Combination of Run-1 exotic searches in diboson final states at the LHC
}

\author{
F. Dias, ${ }^{a}$ S. Gadatsch, ${ }^{b}$ M. Gouzevich, ${ }^{c}$ C. Leonidopoulos, ${ }^{a}$ S.F. Novaes, ${ }^{d}$ A. Oliveira, ${ }^{e}$ \\ M. Pierini ${ }^{b}$ and T. Tomei ${ }^{d}$ \\ ${ }^{a}$ University of Edinburgh, \\ Edinburgh, U.K. \\ ${ }^{b} C E R N$, \\ Geneva, Switzerland \\ ${ }^{c}$ Institut de Physique Nucléaire de Lyon, Université de Lyon, Université Claude Bernard Lyon 1, \\ CNRS-IN2P3, \\ Villeurbanne, France \\ ${ }^{d}$ Universidade Estadual Paulista, \\ Sao Paulo, Brazil \\ ${ }^{e}$ Università di Padova e INFN - Sezione di Padova, \\ Padova, Italy \\ E-mail: Flavia.Dias@cern.ch, Stefan.Gadatsch@cern.ch, \\ Mgouzevi@ipnl.in2p3.fr, Christos.Leonidopoulos@cern.ch, \\ Sergio.Novaes@cern.ch, Alexandra.Oliveira@cern.ch, \\ Maurizio.Pierini@cern.ch, Thiago.Tomei@cern.ch
}

ABSTRACT: We perform a statistical combination of the ATLAS and CMS results for the search of a heavy resonance decaying to a pair of vector bosons with the $\sqrt{s}=8 \mathrm{TeV}$ datasets collected at the LHC. We take into account six searches in hadronic and semileptonic final states carried out by the two collaborations. We consider only public information provided by ATLAS and CMS in the HEPDATA database and in papers published in refereed journals. We interpret the combined results within the context of a few benchmark new physics models, such as models predicting the existence of a $\mathrm{W}^{\prime}$ or a bulk RandallSundrum spin-2 resonance, for which we present exclusion limits, significances, $p$-values and best-fit cross sections. A heavy diboson resonance with a production cross section of $\sim 4-5 \mathrm{fb}$ and mass between 1.9 and $2.0 \mathrm{TeV}$ is the exotic scenario most consistent with the experimental results. Models in which a heavy resonance decays preferentially to a WW final state are disfavoured.

KeYwords: Phenomenology of Field Theories in Higher Dimensions

ARXIV EPRINT: 1512.03371 


\section{Contents}

1 Introduction 1

2 General methodology 4

3 Fully hadronic searches: VV $\rightarrow$ JJ $\quad 5$

3.1 Emulation of ATLAS search 6

3.1.1 Description of the ATLAS analysis 6

$\begin{array}{lll}3.1 .2 & \text { Statistical analysis } & 6\end{array}$

3.1.3 Results with WW, WZ and ZZ signal hypotheses $\quad 7$

$\begin{array}{ll}3.2 & \text { Emulation of CMS search }\end{array}$

3.2.1 Description of the CMS analysis 9

$\begin{array}{lll}3.2 .2 & \text { Statistical analysis } & 10\end{array}$

$\begin{array}{lll}3.3 & \text { Combined LHC results of hadronic searches } & 10\end{array}$

4 Semi-leptonic searches: $\mathrm{WV} \rightarrow \ell \nu \mathrm{J}$ and ZV $\rightarrow \ell \ell \mathrm{J}$

$\begin{array}{ll}4.1 \text { Emulation of ATLAS search } & 15\end{array}$

$\begin{array}{lll}\text { 4.1.1 Description of the ATLAS analysis } & 15\end{array}$

$\begin{array}{lll}\text { 4.1.2 Statistical analysis } & 15\end{array}$

$\begin{array}{lll}4.2 & \text { Emulation of CMS search } & 16\end{array}$

$\begin{array}{lll}\text { 4.2.1 Description of the CMS analysis } & 16\end{array}$

$\begin{array}{lll}4.2 .2 & \text { Statistical analysis } & 17\end{array}$

4.3 Combined LHC results of semi-leptonic searches 22

5 Combination of hadronic and semi-leptonic channels 24

$\begin{array}{llr}6 & \text { Conclusions } & 27\end{array}$

A Comparison of different approaches to emulate ATLAS VV $\rightarrow$ JJ analysis $\quad 29$

$\begin{array}{ll}\text { B Narrow width approximation } & 31\end{array}$

\section{Introduction}

Searches for new heavy resonances are one of the major components of the ATLAS and CMS physics programmes at the Large Hadron Collider (LHC) at CERN. Of particular interest is the coupling of new resonances to pairs of vector bosons. Models with Vectorial heavy resonances (i.e. $\mathrm{W}^{\prime}$-like and $\mathrm{Z}^{\prime}$-like bosons) are commonly considered as possible extensions of the SM, either in weakly coupled (see [1-3]) or strongly coupled versions, the so-called 
composite Higgs scenarios [4, 5]. In these scenarios, the existence of new resonances is introduced to alleviate the hierarchy problem in the SM. Another common SM extension is the Warped Extra Dimensions or Randall-Sundrum (RS) model [6], which is an example of a class of models predicting neutral spin-2 resonances as Kaluza-Klein (KK) excitations of the graviton field $\left(G^{*}\right)$. Two types of models are usually considered: the original version, in which only gravity is allowed to propagate into the extra-dimensional bulk ("RS1" models, see ref. [7]) and variants of the original model, in which the SM fields are also allowed to propagate into the extra dimensional bulk ("bulk RS" models, see for example ref. [8]). RS1 models favour the decay of $G^{*}$ to $q \bar{q}, \ell^{+} \ell^{-}$and $\gamma \gamma$ final states, whereas in bulk RS models its decay to vector bosons.

After a number of direct and indirect bounds from previous experiments, and in particular, the stringent constraints from the electro-weak precision measurements carried out at LEP $[9],{ }^{1}$ nowadays searches for heavy exotic resonances decaying to pairs of vector bosons typically focus on resonance masses above $1 \mathrm{TeV}$. When produced and decayed at the LHC, these particles would generate vector bosons with $\mathcal{O}(1 \mathrm{TeV})$ transverse momenta, requiring special reconstruction strategies. In particular, the quarks from a hadronicallydecaying vector boson are very close to each other in the $\eta-\phi$ space. In their showering and hadronisation process they produce highly overlapping jets, in a so-called boosted topology. ATLAS and CMS handle this experimental signature by reconstructing the two partially overlapping jets as a single massive (or "fat") jet, noted in this paper as "J". One then exploits the jet mass $m_{J}$ and the momentum flow around the jet axis to distinguish these special jets from those originating from quark or gluon production [12-17]. A typical boosted longitudinally polarised and hadronically-decaying $\mathrm{V}$ boson, ${ }^{2}$ can be identified by a tagger with an efficiency of $\sim 50 \%$ and with a false-positive rate for light quarks or gluons of $\lesssim 2 \%[18,19]$.

The ATLAS and CMS collaborations have employed hadronic boson taggers in searches for heavy resonances in diboson final states with the proton-proton collision data collected in 2012 at a centre-of-mass energy of $8 \mathrm{TeV}$. In particular, the ATLAS search in the fully hadronic final state [20] has generated significant interest due to an excess of diboson events with invariant mass mass around $1.9 \mathrm{TeV}$. Small deviations in the same mass region are observed in other channels as well, e.g. the CMS search in the $Z\left(\ell^{+} \ell^{-}\right) V(q \bar{q})$ channel with $\ell=e, \mu[21]$, and the CMS search in the fully hadronic $V(q \bar{q}) V(q \bar{q})$ final state [22]. Other analyses, e.g. the ATLAS and CMS searches in the $W(\ell \nu) V(q \bar{q})$ channel see no evidence of a deviation, indicating a possible tension between these experimental results in the scenario of a heavy exotic resonance. Additional results with potentially interesting deviations in the same mass region include a moderate excess $(\approx 1-2 \sigma$ of local significance) reported in the ATLAS [23, 24] and CMS [25, 26] searches in the dijet channel, as well as in the CMS search in the dilepton channel [27]. In addition, a search for right-handed $\mathrm{W}^{\prime}$ (and heavy neutrinos) [28] by CMS has reported a small excess in the electron channel [29] (however, this excess is not confirmed by a similar ATLAS analysis [30]). Finally, a CMS search

\footnotetext{
${ }^{1}$ For recent analyses, including the LHC discovery of the Higgs boson, see for instance [10, 11].

${ }^{2}$ In this paper we refer to a vector boson ( $\mathrm{W}$ or $\mathrm{Z}$ ) decaying hadronically by the generic label $\mathrm{V}$.
} 
for $\mathrm{W}(\ell \nu) \mathrm{H}(b \bar{b})$ resonances reported an excess of $\approx 2 \sigma$, originating from a stronger excess in the electron channel and no evidence of a deviation in the muon channel [31]. At the same time, the CMS searches for $\mathrm{WH}$ or $\mathrm{ZH}$ resonances in the fully hadronic channel were inconclusive, with a mild upward fluctuation around $1.8 \mathrm{TeV}$ and a lack of events around $2 \mathrm{TeV}$ [32]. The dedicated searches for $\mathrm{Z}(q \bar{q}) \mathrm{H}\left(\tau^{+} \tau^{-}\right)$and $\mathrm{H}(b \bar{b}) \mathrm{H}\left(\tau^{+} \tau^{-}\right), \mathrm{H}(b \bar{b}) \mathrm{H}(b \bar{b})$ final states showed no excess [33, 34].

Several attempts to provide a possible interpretation for this excess have been made during the last months. The deviation has been associated to possible signatures of various beyond-the-SM models, e.g. models with new $W^{\prime}$ and $Z^{\prime}$ vector bosons (see for example [35-44]), models involving new resonances with different spins (see for example [45-52]), composite and technicolor models (see for example [53-58]) and new and composite Higgs states (see for example [59-67]). A review of the different models offering an interpretation of the deviations reported in the ATLAS and CMS searches has been made in ref. [68].

A natural next step would be to carry out a systematic comparison of the results reported by ATLAS and CMS in various channels, and examine if the apparent deviations work in a synergistic way towards a coherent picture. In particular, the goal is to quantify the level of agreement among the different results, and by using an exotic signal hypothesis for the interpretation of these deviations, to calculate the corresponding production cross section. We hereby present the first step in addressing this question, starting with the statistical combination of the results of the ATLAS and CMS Run-1 searches for vector boson pair resonances. The exotic models considered by the experiments are usually connected with the electroweak sector, with the predicted resonances mainly coupling to longitudinally polarised vector bosons $\mathrm{V}_{\mathrm{L}}$. We consider the experimental results of the searches for heavy resonances decaying to three final states: $\mathrm{Z}_{\mathrm{L}} \mathrm{Z}_{\mathrm{L}}, \mathrm{W}_{\mathrm{L}} \mathrm{W}_{\mathrm{L}}$ and $\mathrm{W}_{\mathrm{L}} \mathrm{Z}_{\mathrm{L}}$. We combine the results and interpret the derived exclusion limits in the context of a (W'-like) spin-1 charged particle decaying to a $\mathrm{W}_{\mathrm{L}} \mathrm{Z}_{\mathrm{L}}$ boson pair, and a neutral spin-2 particle $\left(\mathrm{G}_{\text {bulk }}\right)$. For the latter case, we only consider bulk RS scenarios, namely particles decaying to the $\mathrm{Z}_{\mathrm{L}} \mathrm{Z}_{\mathrm{L}}$, $\mathrm{W}_{\mathrm{L}} \mathrm{W}_{\mathrm{L}}$ final states. ${ }^{3}$

The paper is organised as follows: in section 2 we present a general overview of the methodology used to emulate the ATLAS and CMS analyses; sections 3 and 4 discuss the emulation of the hadronic and semileptonic analyses, respectively. Each section covers the individual searches by ATLAS and CMS, and their combination; in section 5 we combine the Run-1 results provided by the two collaborations and discuss their interpretation in a few benchmark models considered in this study; we present the summary of the findings, along with the conclusions in section 6 . A brief note on the compatibility of the findings of this study with the preliminary Run-2 search results reported by ATLAS and CMS in December 2015 has been added in v2 of this paper and is presented after the conclusions. Additional information on the determination of the background and signal modelling for the ATLAS search in the fully hadronic channel is given in appendices A, B.

\footnotetext{
${ }^{3}$ Models in which the exotic resonances have stronger couplings to transverse vector bosons $\left(\mathrm{V}_{\mathrm{T}}\right)$ than longitudinal ones $\left(V_{L}\right)$ typically have larger branching fractions to dilepton and dijet final states. It should be noted that boosted boson taggers are more efficient with $\mathrm{V}_{\mathrm{L}}$ than $\mathrm{V}_{\mathrm{T}}$ bosons [18]. This topic will be addressed in a future publication.
} 


\section{General methodology}

All exotic searches considered in this paper are looking for a diboson mass peak emerging on top of a falling background spectrum. In order to evaluate the significance of a deviation observed in the data, we need as input the shapes of the signal and background distributions, the total number of expected background events, the signal efficiency, and the experimentally measured distribution (data).

This study is based exclusively on the public information provided by the two experimental collaborations in the HEPDATA database [69] and the cited papers (published in refereed journals). In particular, we employ the expected backgrounds with their corresponding uncertainties, as they have been estimated directly by ATLAS and CMS, wherever possible. The modelled signal distributions (namely, shapes and signal efficiencies for a few benchmark models and mass values) are also taken from the information publicly provided by the experiments, when available. ${ }^{4}$ In order to emulate signal distributions for additional mass values, we carry out linear interpolations of the available models within the benchmark mass points. We derive exclusion limits on hypothetical signals by performing binned templated fits of the data distributions with linear combinations of the signal and background distributions. These calculations are carried out with the open-source statistical framework THETA [70] which uses the asymptotic approximation [71] of the CLs method $[72,73]$.

In a few cases, the information published by ATLAS and CMS is not sufficient for this simple approach to produce satisfactory results. For example, uncertainty correlations that affect the background determination, or the mass-dependence of an important systematic uncertainty are not always properly documented. In these cases, we fit the data distributions to the functional form documented in the published analysis, e.g. the function used in the hadronic searches or an exponential function for the leptonic channels. Details about these fits are given in the corresponding sections of the paper, where we also discuss the agreement achieved in the background modelling. When it is necessary to model a signal distribution ourselves, we either use a Gaussian approximation with a resolution inferred from the relevant experimental paper, or we generate Monte Carlo (MC) samples using the Madgraph5 matrix-element event generator [74], matched to Pythia8 [75] for the hadronisation process. For the $\mathrm{G}_{\text {bulk }}$ signal we use the Madgraph5 model files as presented in ref. [76], while for the spin-1 signal $\mathrm{W}^{\prime}$ the ones described in ref. [77].

These approximations are mainly motivated by our familiarity with the diboson and similar searches by ATLAS and CMS. The described procedure is validated using the nominal published results as benchmarks, as well as the comparison of our own calculations of the per-experiment combinations against the official combination of diboson searches [21, 78]. We are able to reproduce the exclusion limits of each analysis individually and their combinations with an agreement of better than $20 \%$ in the region of interest for all channels, with the exception of the fully hadronic search in ATLAS (see appendix A). Our methodology

\footnotetext{
${ }^{4}$ The ATLAS and CMS collaborations usually provide the histograms for a signal benchmark model at a fixed mass value. Often, these histograms are not provided in electronic format. In these cases, we had to extract the information from the publicly available plots.
} 


\begin{tabular}{|ccccccc|}
\hline Experiment & Channel & $\begin{array}{c}\text { Background } \\
\text { modelling }\end{array}$ & $\begin{array}{c}\text { Background } \\
\text { uncertainties }\end{array}$ & $\begin{array}{c}\text { Signal } \\
\text { modelling }\end{array}$ & $\begin{array}{c}\text { Signal } \\
\text { efficiency }\end{array}$ & $\begin{array}{c}\text { Fudge } \\
\text { factor }\end{array}$ \\
\hline \multirow{4}{*}{ ATLAS } & JJ $[20]$ & Fit & Fit & Paper \& extrap. & Public plots & Yes \\
& $\ell \nu \mathrm{J}[79]$ & HEPDATA & HEPDATA & Gauss. approx. & Public plots & Yes \\
& $\ell \ell \mathrm{J}[80]$ & Fit & HEPDATA & Gauss. approx. & Public plots & Yes \\
\hline \multirow{3}{*}{ CMS } & JJ $[22]$ & HEPDATA & HEPDATA & HEPDATA & HEPDATA & No \\
& $\ell \nu \mathrm{J}[21]$ & Fit & Fit & MC & Public plots \& MC & Yes \\
& $\ell \ell \mathrm{J}[21]$ & Fit & Fit & MC & Public plots \& MC & Yes \\
\hline
\end{tabular}

Table 1. Summary of the methods used and the corresponding uncertainties for the signal and background modelling per channel and experiment.

can be used as a set of guidelines for model builders in the absence of official combined results published by the two experiments.

All diboson final states considered in this study contain at least one vector boson (W or Z) decaying hadronically. Because of the limited hadronic detector resolution, it is not possible to distinguish between hadronic $\mathrm{W}$ and hadronic $\mathrm{Z}$ jets. When interpreting an experimental result, special care is needed to account for possible cross-channel contamination of the final state under consideration. For example, a neutral heavy resonance decaying to a pair of vector bosons is expected to decay to both $\mathrm{WW}$ and $\mathrm{ZZ}$ final states. We consider models in which the relative branching fractions of neutral particle decays to $\mathrm{WW}$ and ZZ can vary, in order to study the relative importance of the different bosonic sub-channels to the combined result. We quantify this dependence by introducing as a free parameter the ratio $r$ of the corresponding branching fractions:

$$
r \equiv \frac{\mathcal{B}(X \rightarrow \mathrm{WW})}{\mathcal{B}(X \rightarrow \mathrm{ZZ})}
$$

with $r=2$ being the default ratio in the baseline bulk RS scenario.

The full list of channels that we consider in this study is as follows: the fully hadronic searches $X \rightarrow V(q \bar{q}) V(q \bar{q})$ (labelled "JJ"), searches including a W decaying leptonically $X \rightarrow \mathrm{W}(\ell \nu) V(q \bar{q})$ (labelled " $\ell \nu \mathrm{J}$ "), and searches including a $\mathrm{Z}$ decaying leptonically $X \rightarrow$ $\mathrm{Z}(\ell \ell) V(q \bar{q})$ (labelled " $\ell \mathrm{J}$ "). Table 1 summarises the methods that have been used to emulate each of the analyses considered. Details of the individual analyses are given in the sections that follow.

\section{Fully hadronic searches: VV $\rightarrow$ JJ}

In this section we discuss the analysis of the ATLAS and CMS searches in the VV $\rightarrow$ JJ channel. We first present the results of our analysis for the two searches separately, followed by their combination and a summary of the findings. 


\subsection{Emulation of ATLAS search}

\subsubsection{Description of the ATLAS analysis}

The ATLAS fully hadronic search analyses calorimetric dijet events. The main irreducible background is dijet production in QCD, which is dominated by $2 \rightarrow 2 t$-channel processes involving quarks and gluons. The contribution of these processes is minimised by restricting the jet acceptance to $|\eta|<2.0$ and the rapidity difference between those two jets to $|\Delta \eta|<$ 1.2. The events are required to have low missing transverse momentum and a rather symmetric dijet topology (similar $p_{\mathrm{T}}$ for the two leading jets) to reduce the detector noise. After this selection, the efficiency is approximately $70-80 \%$ for a heavy vector boson signal, and above $80 \%$ for a $\mathrm{G}_{\text {bulk }}$ signal.

To further reduce the multijet background, two fat jets are reconstructed using the Cambridge-Aachen algorithm [81, 82] with radius parameter $R=1.2$. The mass-drop filtering algorithm [12] is applied to each of these jets for the identification of the subjets and grooming. Events are kept if each of the two leading jets satisfies the following conditions: have two sub-jets with similar transverse momentum, have less than 30 tracks matched to it, and have a pruned mass within a $\pm 13 \mathrm{GeV}$ window either around $82.4 \mathrm{GeV}$ (for $\mathrm{W}$ tagging) or around $92.8 \mathrm{GeV}$ (for $\mathrm{Z}$ tagging). The selection efficiency of the grooming algorithm for fat jets from a $\mathrm{W}^{\prime}$ resonance is between $30 \%$ and $40 \%$.

The events are subsequently classified into three non-mutually-exclusive categories, based on the jet-mass values: WW, WZ and ZZ. The overall product of the geometric acceptance with the signal efficiency for this analysis is typically $10-20 \%$.

\subsubsection{Statistical analysis}

The analysis uses the smoothness test ("bump search") approach: the background is approximated by a steeply falling function, while the signal template is taken from simulation. The sum of the two components is then fitted to the data. The background function used by the ATLAS collaboration is:

$$
f\left(m_{\mathrm{JJ}}\right)=p_{0}\left(1-m_{\mathrm{VV}}\right)^{p_{1}-\xi p_{2}} m_{\mathrm{VV}}^{p_{2}}
$$

where $p_{0}, p_{1}$ and $p_{2}$ are free parameters and $m_{\mathrm{JJ}}$ is the dijet invariant mass; ATLAS has also made the signal templates used in the analysis public. We employ the same function for the background description, but recalculate the background uncertainties in order to better account for the large scale correlations in $m_{\mathrm{JJ}}$. To this end, we refit the data in each of the three categories above using the aforementioned background parametrisation. We diagonalise the uncertainty matrix and obtain three uncertainty eigenvectors $\left(\sigma_{\lambda_{i}}\right.$, with $i=0,1,2)$. Our fit result produces a background estimate which agrees with the nominal background within $10 \%$, which is well within the uncertainties (see appendix A). This background is subsequently used together with the associated uncertainties in our statistical analysis (see figure 1).

We consider the following systematic uncertainties, treated as fully correlated across $m_{\mathrm{JJ}}$ histogram bins:

- Background uncertainty, obtained as described above. 

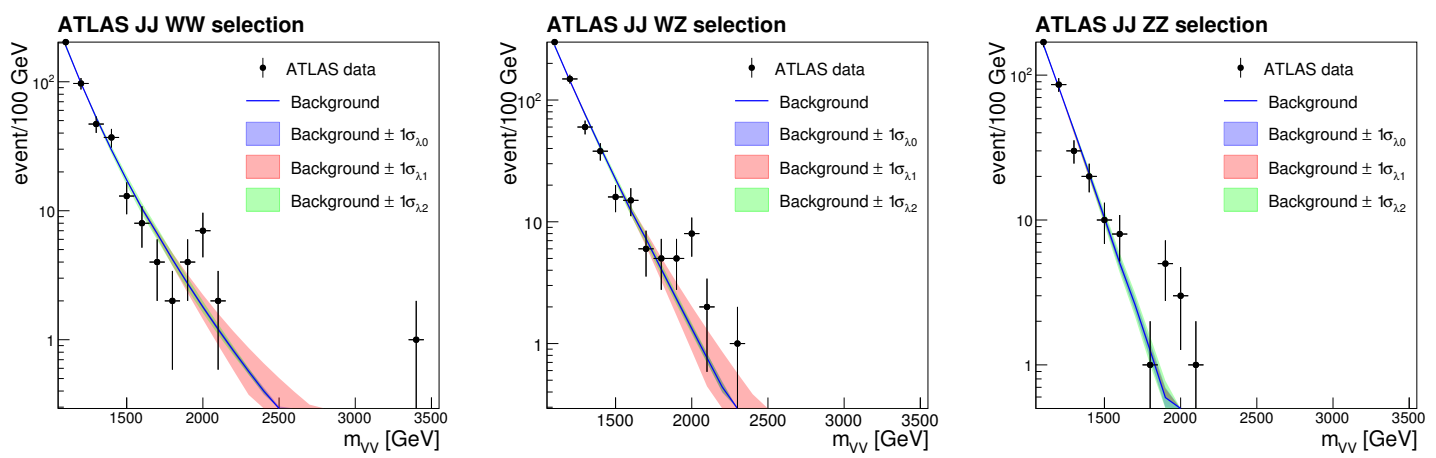

Figure 1. ATLAS hadronic search: comparison between the official ATLAS fit (blue line) and the fit of this study with uncertainties as described in the text (coloured bands), with the overlaid data of the $m_{\mathrm{JJ}}$ spectrum for the WW (left), WZ (middle) and ZZ (right) tagging selections.

- Signal normalisation uncertainty, which is separated into two further sub-categories: a common-across-channels systematic uncertainty corresponding to the luminosity measurement (2.8\%), and an additional term applicable to the JJ channel that covers V-tagging uncertainties as well as jet systematics.

- Signal jet energy scale uncertainty, which includes jet transverse momentum and mass uncertainties (with a $\pm 2 \%$ and $\pm 5 \%$ impact on $m_{\mathrm{JJ}}$, respectively). An additional jet energy resolution uncertainty is known to have a negligible effect on the signal shape and is ignored in this study.

Our statistical analysis produces expected exclusion limits that are typically $50 \%$ more stringent than the ones publicly provided by ATLAS. This discrepancy, discussed in detail in appendix A, is corrected for with the introduction of a fudge factor, defined as the ratio of the ATLAS expected exclusion limits and the ones from this study obtained with the THETA statistical framework (see figure 2). With this correction, our calculated exclusion limits are in good agreement with the public ATLAS results (see figure 3).

\subsubsection{Results with WW, WZ and ZZ signal hypotheses}

As discussed above, due to the finite detector resolution, the $\mathrm{V}$-tagging tool is not capable to differentiate between fat jets originating from $\mathrm{W}$ or $\mathrm{Z}$ bosons. However, there is a significant performance difference between $\mathrm{W}$ and $\mathrm{Z}$ tagging efficiencies of up to $\approx 30 \%$, mainly as a result of the different boson masses. By using the mass distribution of longitudinal Vjets, as documented in figure 1 of ref. [20], and by taking into account the different $\mathrm{W}$ and $\mathrm{Z}$ efficiencies, we can calculate the efficiency of tagging selections for different signal hypotheses (WW, WZ, ZZ). The comparison of the tagging selection efficiencies can be found in table 2 .

The effect of applying the different tagging selections to the WW, WZ and ZZ signal hypotheses as a function of the resonance mass is shown in figure 4 . We assume that the $m_{\mathrm{JJ}}$ spectrum is not affected by the mass window difference in the tagging selections, i.e. that the same distribution describes the three tagging categories WW, WZ and ZZ. 


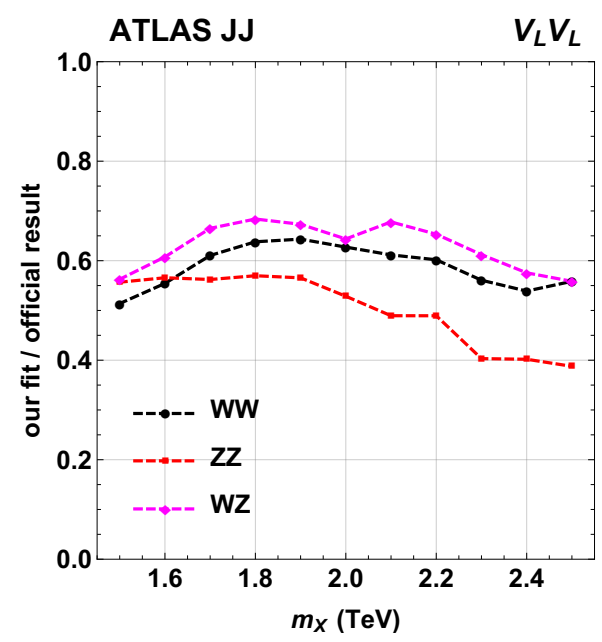

Figure 2. ATLAS hadronic search: ratio of observed exclusion limits obtained with this study to the ones of the official ATLAS result, as a function of the mass $m_{X}$ of the exotic resonance for the WW (black), ZZ (red) and WZ (magenta) tagging selections.
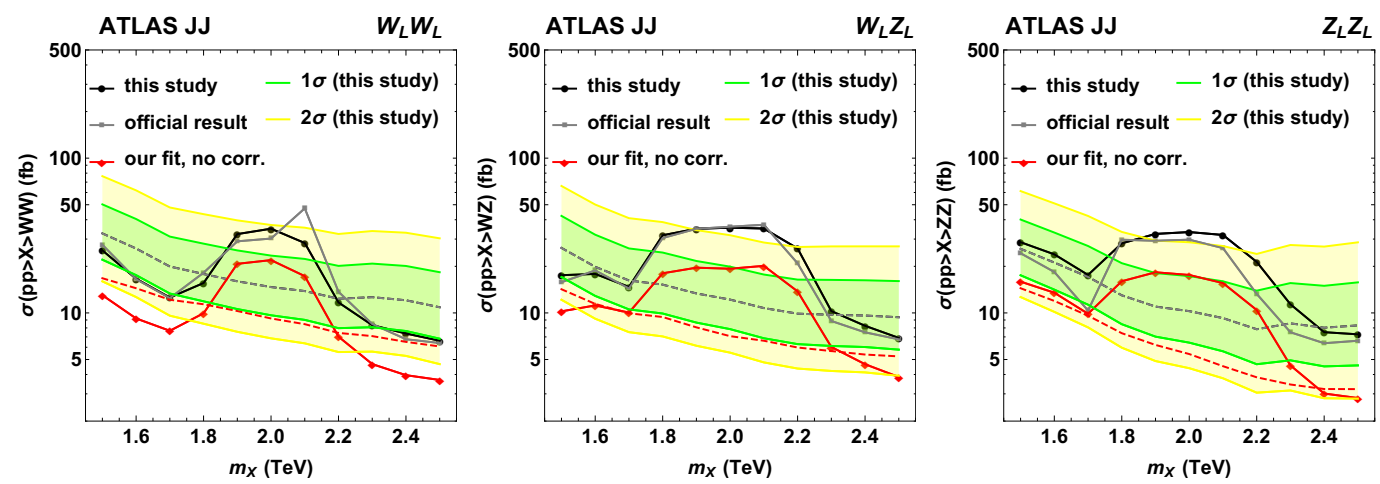

Figure 3. ATLAS hadronic search: observed exclusion limits on exotic production cross section as a function of the resonance mass $m_{X}$ obtained with this study, with (black) and without (red) the correction discussed in the text ("fudge"), and comparison with the official ATLAS results (grey) for $\mathrm{G}_{\text {bulk }} \rightarrow \mathrm{W}_{L} \mathrm{~W}_{L}$ (left), $\mathrm{W}^{\prime} \rightarrow \mathrm{W}_{L} \mathrm{Z}_{L}$ (middle) and $\mathrm{G}_{\text {bulk }} \rightarrow \mathrm{Z}_{L} \mathrm{Z}_{L}$ (right) signal hypotheses and tagging selections. The green and yellow bands represent the one and two sigma variations around the median expected limits (dashed lines) calculated with the same fudge factor.

\begin{tabular}{|cccc|}
\hline & \multicolumn{3}{c|}{ Signal hypothesis } \\
\cline { 2 - 4 } Tagging selection & WW & WZ & ZZ \\
\hline WW window & 1.00 & 0.65 & 0.42 \\
WZ window & 0.84 & 1.00 & 0.65 \\
ZZ window & 0.70 & 0.84 & 1.00 \\
\hline
\end{tabular}

Table 2. Relative efficiencies for WW, WZ, ZZ signal hypotheses for tagging selection using different mass windows. 

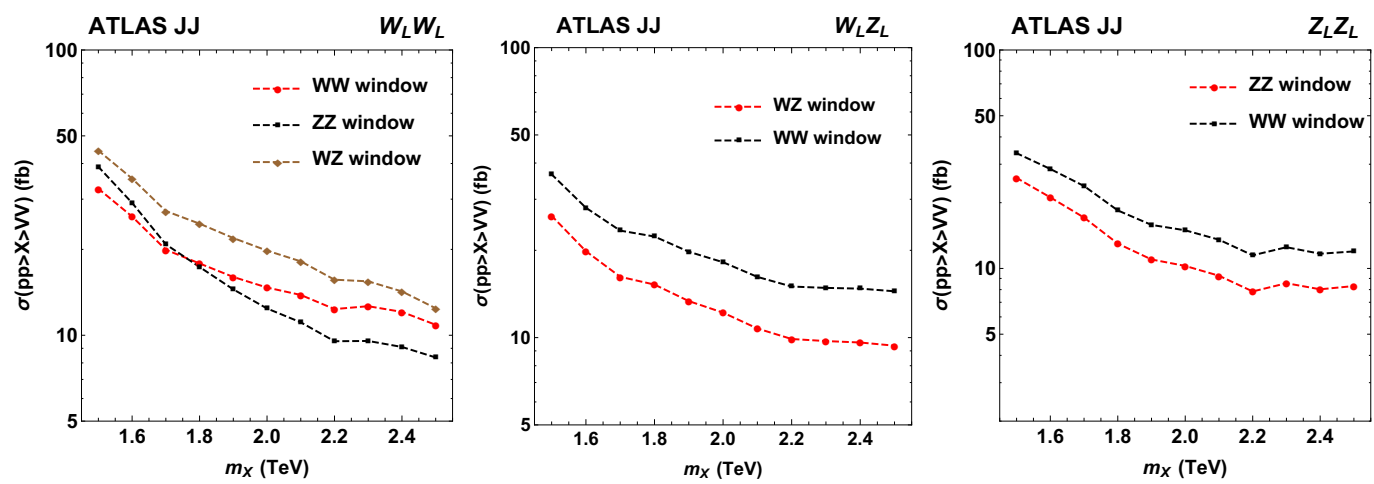

Figure 4. ATLAS hadronic search: expected exclusion limits for different tagging and masswindow selections, as a function of the mass $m_{X}$ of the exotic resonance for $\mathrm{G}_{\mathrm{bulk}} \rightarrow \mathrm{W}_{L} \mathrm{~W}_{L}$ (left), $\mathrm{W}^{\prime} \rightarrow \mathrm{W}_{L} \mathrm{Z}_{L}$ (middle) and $\mathrm{G}_{\text {bulk }} \rightarrow \mathrm{Z}_{L} \mathrm{Z}_{L}$ (right) signal hypotheses. The results have been obtained with the correction discussed in the text.

Since the three categories have common events, they cannot be combined as if they were statistically independent. Instead, for each theoretical model under consideration we choose the tagging category that gives the best expected exclusion limits. For the $\mathrm{W}^{\prime}$ model the WZ tagging selection gives the best result, whereas for the $G_{\text {bulk }}$ graviton model in the $\mathrm{W}_{\mathrm{L}} \mathrm{W}_{\mathrm{L}}$ and $\mathrm{Z}_{\mathrm{L}} \mathrm{Z}_{\mathrm{L}}$ final states the $\mathrm{ZZ}$ tagging selection has the best performance.

\subsection{Emulation of CMS search}

\subsubsection{Description of the CMS analysis}

The jet acceptance is restricted to $|\eta|<2.5$ and $|\Delta \eta|<1.3$ in order to reduce the contamination from multijet events. The detector noise is removed by requiring tight quality criteria on the jets.

The pruning algorithm [13] is used to clean up the jet from soft and large-angle radiation. The mass of the resulting fat jet is constrained in the $70<m_{J}<100 \mathrm{GeV}$ range. Finally, the signal-to-background ratio is enhanced by exploiting the jet $N$-subjettiness [14-16] variable $\tau_{N}$. This variable is used to quantify how well the jet constituents can be arranged into $\mathrm{N}$ sub-jets, i.e. in a consistency check with the hadronic $\mathrm{V}$ boson hypothesis. The ratio $\tau_{12}=\tau_{2} / \tau_{1}$ is built with the two leading jets: the smaller the ratio, the larger the probability that the jet consists of two sub-jets. The analysis considers two categories: the high purity (HP) one, defined by requiring $\tau_{12}<0.5$ for both jets, and the low purity (LP) one, defined by requiring one jet with $\tau_{12}<0.5$ and the other one with $0.5<\tau_{12}<0.75$. The HP category is characterised by a smaller background contamination. The LP category captures signal events with asymmetric decays of the vector-boson candidates in the laboratory frame. Dividing the event sample into the LP and HP categories improves the sensitivity of the analysis in the mass range between $1 \mathrm{TeV}$ and $2 \mathrm{TeV}$, while avoiding the inefficiency of a tight $\tau_{12}$ selection at large jet momenta.

The product of the geometrical acceptance with the signal efficiency is similar to the one in the ATLAS search, ranging between $10 \%$ and $20 \%$. 


\subsubsection{Statistical analysis}

The CMS collaboration provides the binned data and background distributions with the associated uncertainties in the HEPDATA database (see figure 5), as well as the signal distributions for three different models along with their efficiencies [22]: $\mathrm{W}^{\prime} \rightarrow \mathrm{W}_{L} \mathrm{Z}_{L}$ and $\mathrm{G}_{\text {bulk }}$ decaying exclusively to $\mathrm{Z}_{\mathrm{L}} \mathrm{Z}_{\mathrm{L}}$ or $\mathrm{W}_{\mathrm{L}} \mathrm{W}_{\mathrm{L}}$. We consider the following systematic uncertainties:

- Background uncertainty, provided by CMS (in HEPDATA) and considered as fully correlated across the bins of the $m_{\mathrm{JJ}}$ distribution.

- Signal normalisation uncertainty, which is separated further into two sub-categories: a common-across-channels systematic uncertainty corresponding to the luminosity measurement $(2.2 \%)$, and an additional term applicable to the JJ channel that covers V-tagging uncertainties, such as $p_{\mathrm{T}}$, pile-up and PDF dependencies (13\%). The $\tau_{12}$ uncertainties are treated separately in the category below.

- Signal purity category migration uncertainty, which covers the effects of events "migrating" from the HP to the LP category, or vice-versa. This uncertainty amounts to $7.5 \%$ and $54 \%$, respectively.

- Signal jet energy scale uncertainty, propagates to $\pm 1 \%$ of uncertainty on $m_{\mathrm{JJ}}$; it is treated in the same way as in the ATLAS case.

All systematic uncertainties are treated as fully correlated across different $m_{\mathrm{JJ}}$ bins. They are also considered as fully correlated between the LP and the HP categories, with the exception of the "purity category migration" uncertainty, which is treated as fully anticorrelated.

Our statistical analysis for $\mathrm{W}^{\prime} \rightarrow \mathrm{W}_{L} \mathrm{Z}_{L}, \mathrm{G}_{\text {bulk }} \rightarrow \mathrm{W}_{L} \mathrm{~W}_{L}$ and $\mathrm{G}_{\text {bulk }} \rightarrow \mathrm{Z}_{L} \mathrm{Z}_{L}$ models produces exclusion limits that are in very good agreement with the ones publicly provided by CMS. An example of this agreement can been seen in the left plot of figure 6 . The exclusion limits calculated in a few benchmark models can be seen in the right plot of figure 6 . The most stringent limits are obtained for the $\mathrm{G}_{\text {bulk }} \rightarrow \mathrm{Z}_{L} \mathrm{Z}_{L}$ hypothesis, thanks to the higher $\mathrm{V}$-tagging efficiency for $\mathrm{Z}$ bosons.

\subsection{Combined LHC results of hadronic searches}

This section describes the combination of the ATLAS and CMS searches in the fully hadronic channel JJ and the interpretation of the results under different signal hypotheses.

As a first step we note that ATLAS assumes a wide resonance in its JJ searches, whereas CMS assumes a narrow one. To ensure a consistent treatment of the search in the hadronic channel between the two experiments we introduce a $+10 \%$ scale factor in the ATLAS selection efficiency. A description of the derivation of the scale factor and its impact on the search sensitivity is discussed in appendix B. For every signal hypothesis under consideration we use the optimal mass selection windows as defined by ATLAS.

We proceed by combining the THETA data cards of the individual ATLAS and CMS searches. The results of the statistical combination for the $\mathrm{W}_{\mathrm{L}} \mathrm{Z}_{\mathrm{L}}, \mathrm{W}_{\mathrm{L}} \mathrm{W}_{\mathrm{L}}$, and $\mathrm{Z}_{\mathrm{L}} \mathrm{Z}_{\mathrm{L}}$ 

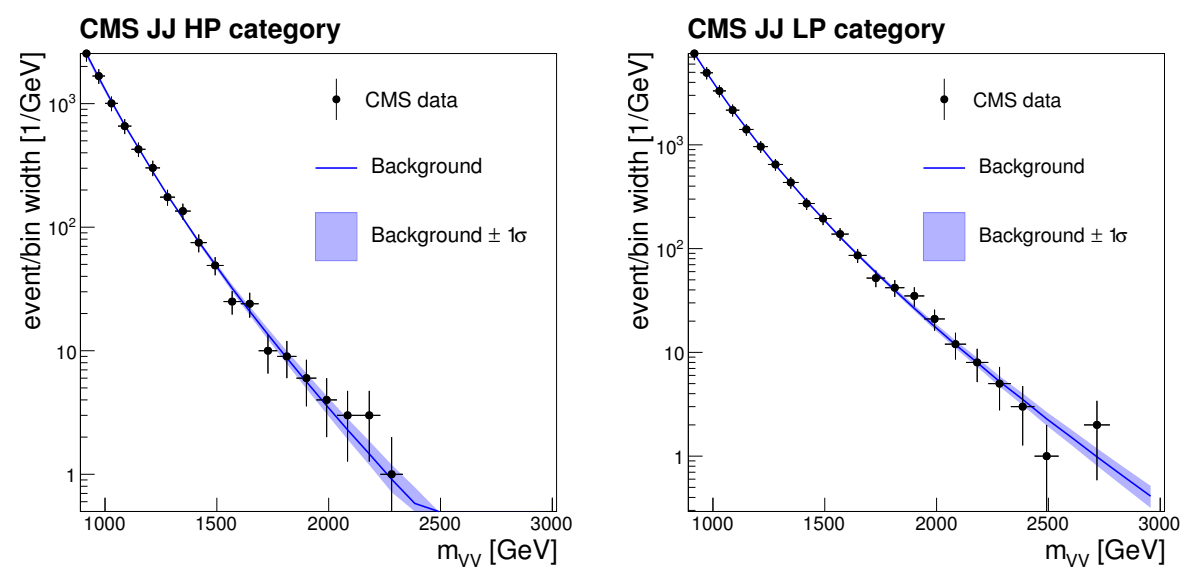

Figure 5. CMS hadronic search: $m_{\mathrm{JJ}}$ data distribution overlaid with the background fit employed in this study with uncertainties for High (left) and Low (right) Purity samples. See text for details.
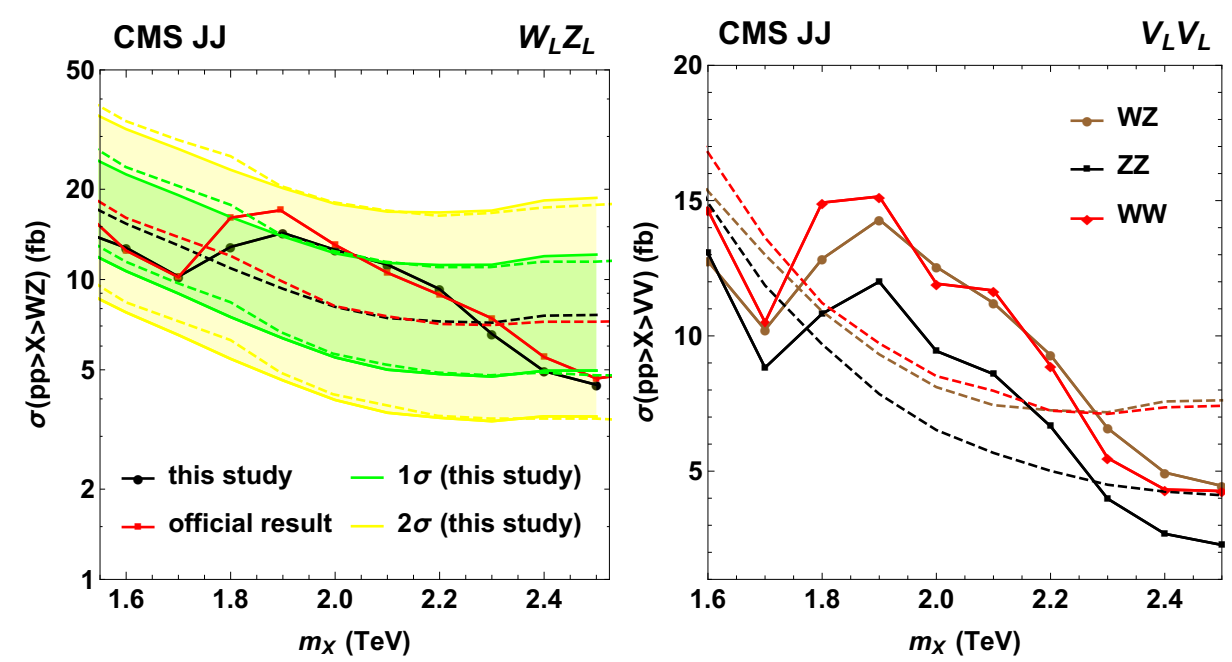

Figure 6. CMS hadronic search. Left: expected (dashed lines) and observed (continuous lines) exclusion limits on $\mathrm{W}^{\prime} \rightarrow \mathrm{W}_{L} \mathrm{Z}_{L}$ production cross sections as a function of the resonance mass $m_{X}$ obtained with this study (black), and comparison with the official CMS results (red). The green and yellow bands (dashed lines) represent the one and two sigma variations around the median expected limits calculated in this study (by CMS). Right: expected (dashed lines) and observed (continuous lines) exclusion limits on exotic production cross section as a function of the resonance mass $m_{X}$ obtained with this study for $\mathrm{W}^{\prime} \rightarrow \mathrm{W}_{L} \mathrm{Z}_{L}$ (brown), $\mathrm{G}_{\text {bulk }} \rightarrow \mathrm{W}_{L} \mathrm{~W}_{L}$ (red) and $\mathrm{G}_{\text {bulk }} \rightarrow \mathrm{Z}_{L} \mathrm{Z}_{L}$ (black) signal hypotheses.

signal hypotheses can be seen in figure 7 . In the $1.7<m_{\mathrm{X}}<2.2 \mathrm{TeV}$ region we observe the largest discrepancy between expected and observed exclusion limits due to the presence of the excess in the $m_{\mathrm{JJ}}$ spectrum. The excess is much smaller in the CMS analysis, which forces the combined results to lie between the ATLAS and the CMS curves. The sensitivity of the combined search as we move away from the deviation region is driven by the CMS analysis. 

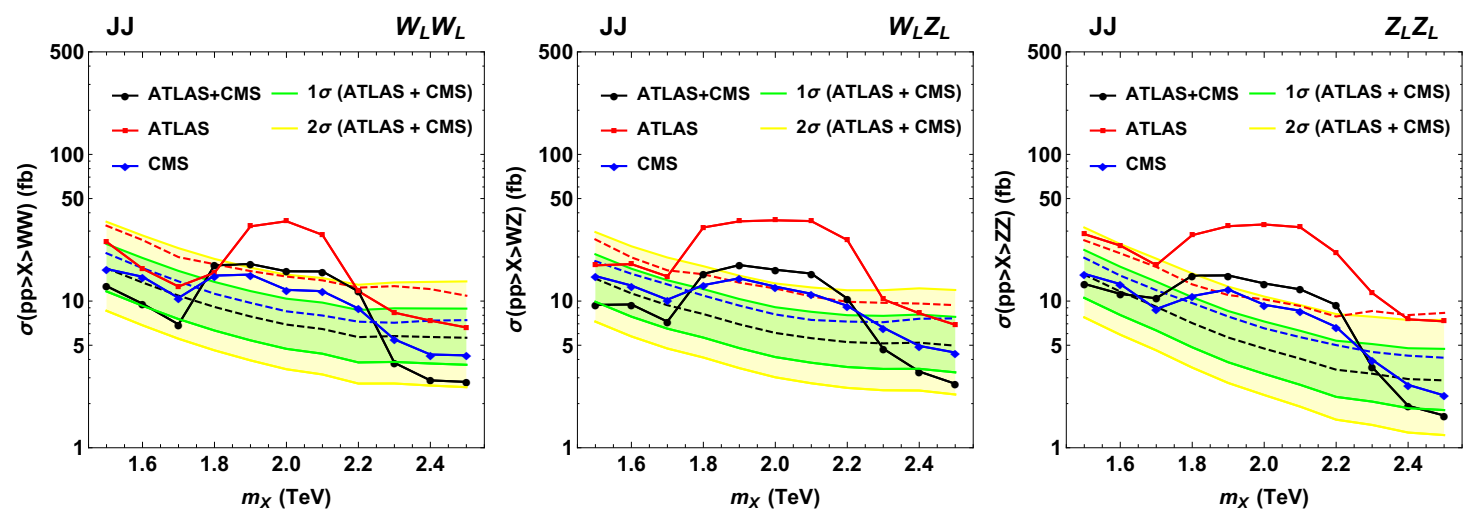

Figure 7. Combination of hadronic searches: expected (dashed lines) and observed (continuous lines) exclusion limits on exotic production cross section as a function of the resonance mass $m_{\mathrm{X}}$ obtained with the emulation of the ATLAS (red) and CMS (blue) searches and their combination (black) for $\mathrm{W}_{\mathrm{L}} \mathrm{W}_{\mathrm{L}}$ (left), $\mathrm{W}_{\mathrm{L}} \mathrm{Z}_{\mathrm{L}}$ (middle) and $\mathrm{Z}_{\mathrm{L}} \mathrm{Z}_{\mathrm{L}}$ (right) selections and signal hypotheses. The green and yellow bands represent the one and two sigma variations around the median expected limits. The results include the $10 \%$ scale factor discussed in the text.

The impact of the individual experimental results on the combination can be seen in the distribution of $p$-values (obtained using Wilks' theorem) depicted in figure 8. The CMS $z$-value or significance ${ }^{5}$ in the excess region is of the order of $1 \sigma$, independently of the considered model and corresponding selections. The ATLAS significance ranges from less than $3 \sigma$ for the $\mathrm{W}_{\mathrm{L}} \mathrm{W}_{\mathrm{L}}$ selection to nearly $4 \sigma$ for the $\mathrm{Z}_{\mathrm{L}} \mathrm{Z}_{\mathrm{L}}$ selection, as a result of the different $\mathrm{W}$ and $\mathrm{Z}$ mass selection windows. The statistical significance of the combined result is very close to the one obtained with the ATLAS result alone, although slightly reduced. In fact, the ATLAS and CMS results are not contradictory: due to the small CMS excess observed in the same mass region, the CMS result cannot exclude the larger ATLAS excess.

In order to further characterise the interplay between the ATLAS and the CMS results in the combination, we show in figure 9 the best-fit exotic signal cross section as a function of the resonance mass $m_{X}$ value for a few benchmark models and corresponding selections: $\mathrm{W}_{\mathrm{L}} \mathrm{Z}_{\mathrm{L}}, \mathrm{W}_{\mathrm{L}} \mathrm{W}_{\mathrm{L}}$ and $\mathrm{Z}_{\mathrm{L}} \mathrm{Z}_{\mathrm{L}}$. The best-fitted cross section values are shown separately for the emulation of ATLAS and CMS searches, and their combination. The largest excess for the $\mathrm{W}_{\mathrm{L}} \mathrm{Z}_{\mathrm{L}}$ and $\mathrm{W}_{\mathrm{L}} \mathrm{W}_{\mathrm{L}}$ signal hypotheses is observed in the $1.9<m_{\mathrm{X}}<2.1 \mathrm{TeV}$ mass range, while the excess extends down to $m_{\mathrm{X}}=1.8 \mathrm{TeV}$ for the $\mathrm{Z}_{\mathrm{L}} \mathrm{Z}_{\mathrm{L}}$ signal hypothesis. In these mass ranges, the ATLAS data suggests a production cross section of $\approx 10 \mathrm{fb}$, whereas the CMS data favours smaller values $(\approx 3 \mathrm{fb})$ and is more consistent with the no-signal hypothesis. The $m_{\mathrm{X}}$ profile of the fitted exotic signal cross section is essential identical to the one obtained from the ATLAS search emulation.

\footnotetext{
${ }^{5}$ The statistics community tends to use the term $z$-value or $z$-score, whereas the physics community prefers to use the term significance.
} 

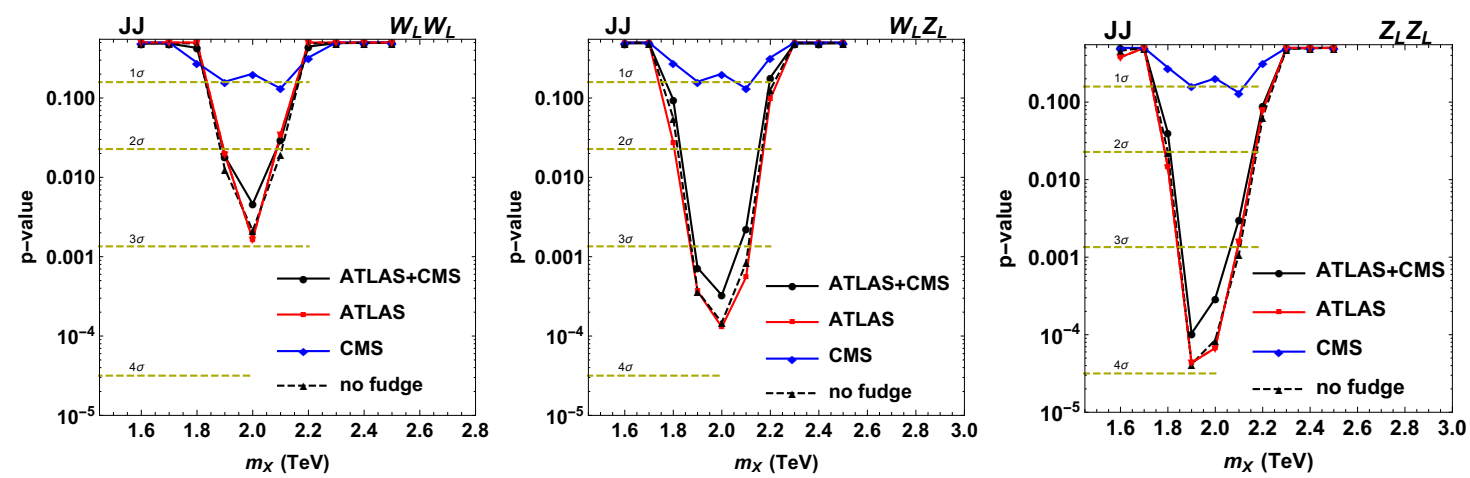

Figure 8. Combination of hadronic searches: likelihood ratio $p$-values as a function of the exotic resonance mass $m_{\mathrm{X}}$ obtained with the emulation of the ATLAS (red) and CMS (blue) searches and their combination (continuous black) for $\mathrm{W}_{\mathrm{L}} \mathrm{W}_{\mathrm{L}}$ (left), $\mathrm{W}_{\mathrm{L}} \mathrm{Z}_{\mathrm{L}}$ (middle) and $\mathrm{Z}_{\mathrm{L}} \mathrm{Z}_{\mathrm{L}}$ (right) selections. The dashed black curve corresponds to the combined search without the $10 \%$ scale factor discussed in the text.
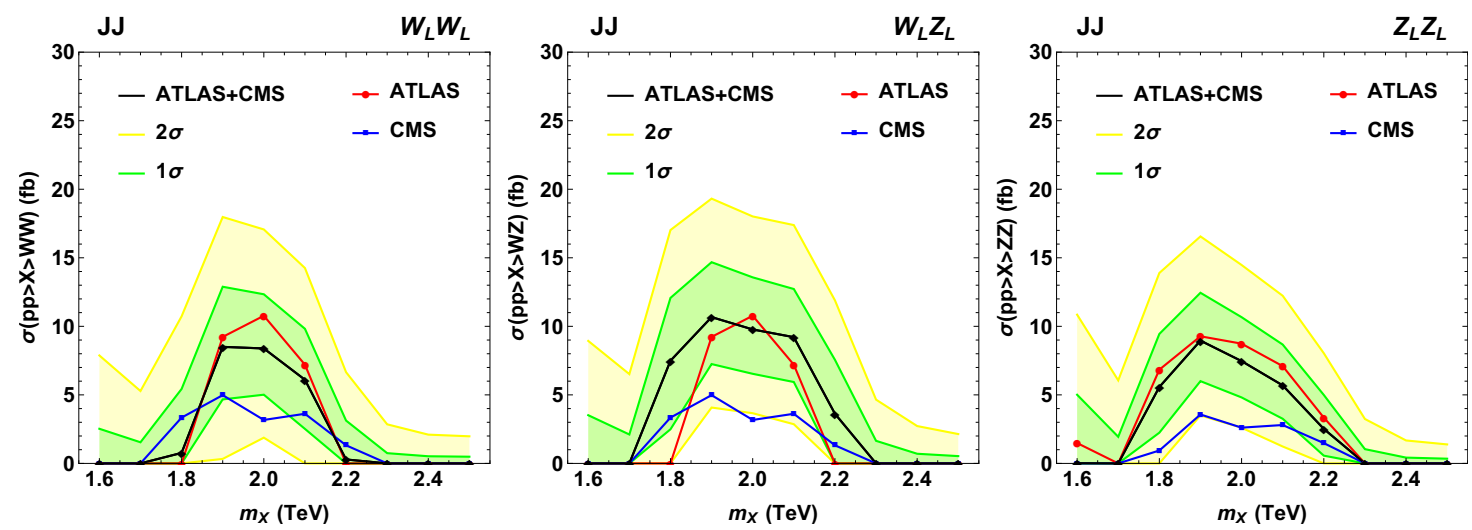

Figure 9. Combination of hadronic searches: best fitted exotic production cross section as a function of the resonance mass $m_{X}$ obtained with the emulation of the ATLAS (red) and CMS (blue) searches and their combination (black) for $\mathrm{W}_{\mathrm{L}} \mathrm{W}_{\mathrm{L}}$ (left), $\mathrm{W}_{\mathrm{L}} \mathrm{Z}_{\mathrm{L}}$ (middle) and $\mathrm{Z}_{\mathrm{L}} \mathrm{Z}_{\mathrm{L}}$ (right) selections and signal hypotheses. The green and yellow bands represent the one and two sigma variations around the median values. The results include the $10 \%$ scale factor discussed in the text.

Further tests of the compatibility between the ATLAS and CMS results can be seen in figure 10, showing scans of the profiled likelihood as a function of the exotic production cross section for $m_{\mathrm{X}}=2 \mathrm{TeV}$ (mass value of largest excess). Due to the large uncertainties of the fit, the best-fit cross-section values by ATLAS and CMS are compatible within $\pm 1 \sigma$ for the $\mathrm{W}_{\mathrm{L}} \mathrm{Z}_{\mathrm{L}}$ and $\mathrm{W}_{\mathrm{L}} \mathrm{W}_{\mathrm{L}}$ hypotheses. The compatibility of the results from the two experiments is slightly reduced in the $\mathrm{Z}_{\mathrm{L}} \mathrm{Z}_{\mathrm{L}}$ scenario. The dependence of these results on $r \equiv \mathcal{B}\left(X \rightarrow \mathrm{W}_{\mathrm{L}} \mathrm{W}_{\mathrm{L}}\right) / \mathcal{B}\left(X \rightarrow \mathrm{Z}_{\mathrm{L}} \mathrm{Z}_{\mathrm{L}}\right)$ can be seen in figure 11. The conclusions discussed above remain mostly unchanged.

In summary, in the combination of fully hadronic results the small CMS excess results in a slight reduction of the larger ATLAS excess. However, the combined-search statistical 

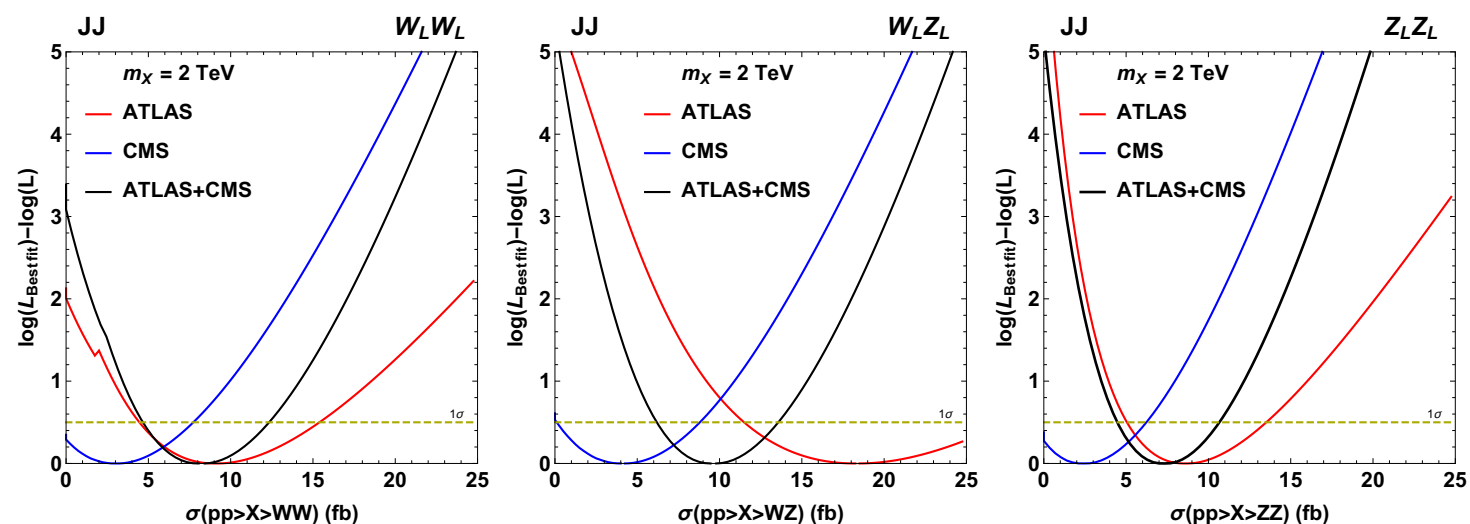

Figure 10. Combination of hadronic searches: scans of the profile likelihood as a function of the exotic production cross section for a $m_{\mathrm{X}}=2 \mathrm{TeV}$ signal (mass value of largest excess) for the emulation of the ATLAS (red) and CMS (blue) searches and their combination (black) for $\mathrm{W}_{\mathrm{L}} \mathrm{W}_{\mathrm{L}}$ (left), $\mathrm{W}_{\mathrm{L}} \mathrm{Z}_{\mathrm{L}}$ (middle) and $\mathrm{Z}_{\mathrm{L}} \mathrm{Z}_{\mathrm{L}}$ (right) selections and signal hypotheses.
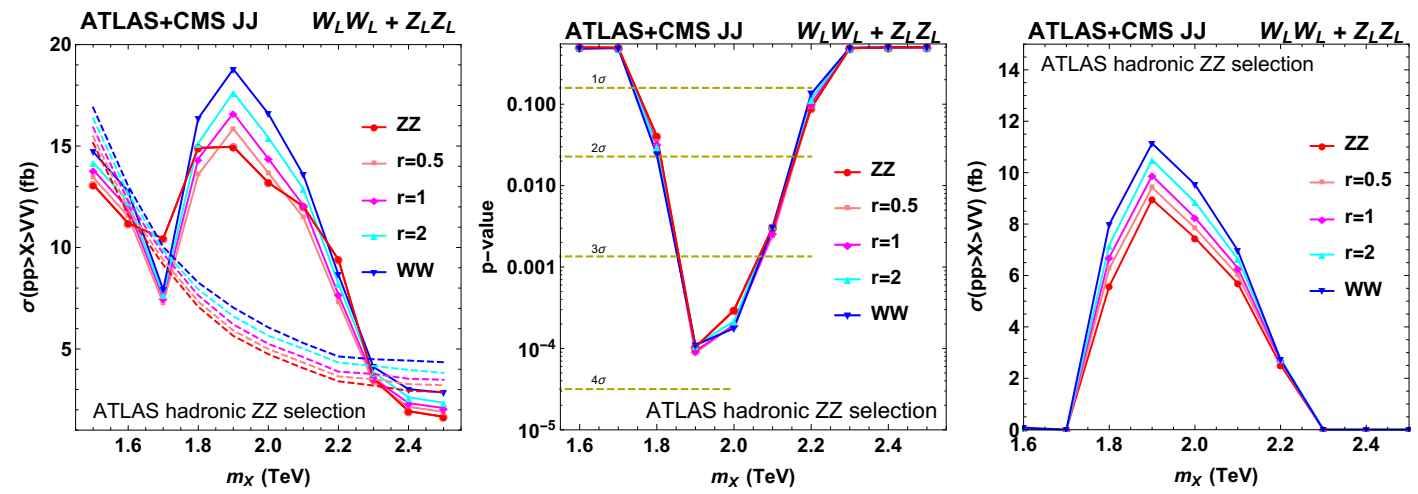

Figure 11. Combination of hadronic searches, and dependence of results obtained in this study on the $r \equiv \mathcal{B}(X \rightarrow \mathrm{WW}) / \mathcal{B}(X \rightarrow \mathrm{ZZ})$ parameter for a neutral bulk RS-like spin-2 particle hypothesis, and as a function of the resonance mass $m_{\mathrm{X}}$. Left: expected (dashed lines) and observed (continuous lines) exclusion limits on exotic production cross section. Middle: likelihood-ratio $p$-values. Right: best fitted exotic production cross section.

significance stays well above $3 \sigma$ for the $\mathrm{W}_{\mathrm{L}} \mathrm{Z}_{\mathrm{L}}$ and $\mathrm{Z}_{\mathrm{L}} \mathrm{Z}_{\mathrm{L}}$ hypotheses and close to $3 \sigma$ for the $\mathrm{W}_{\mathrm{L}} \mathrm{W}_{\mathrm{L}}$ hypotheses. The preferred mass range for a hypothetical exotic signal is 1.9 $<m_{\mathrm{X}}<2.0 \mathrm{TeV}$, with the corresponding production cross section in the $8-12 \mathrm{fb}$ region.

\section{Semi-leptonic searches: WV $\rightarrow \ell \nu \mathrm{J}$ and ZV $\rightarrow \ell \ell J$}

In this section we discuss the analysis of the ATLAS and CMS searches in the WV $\rightarrow \ell \nu \mathrm{J}$ and ZV $\rightarrow \ell \ell J$ channels. We follow the discussion pattern of the fully hadronic section: we first present the results of our analysis for the two searches separately, followed by their combination and a summary of our findings. 

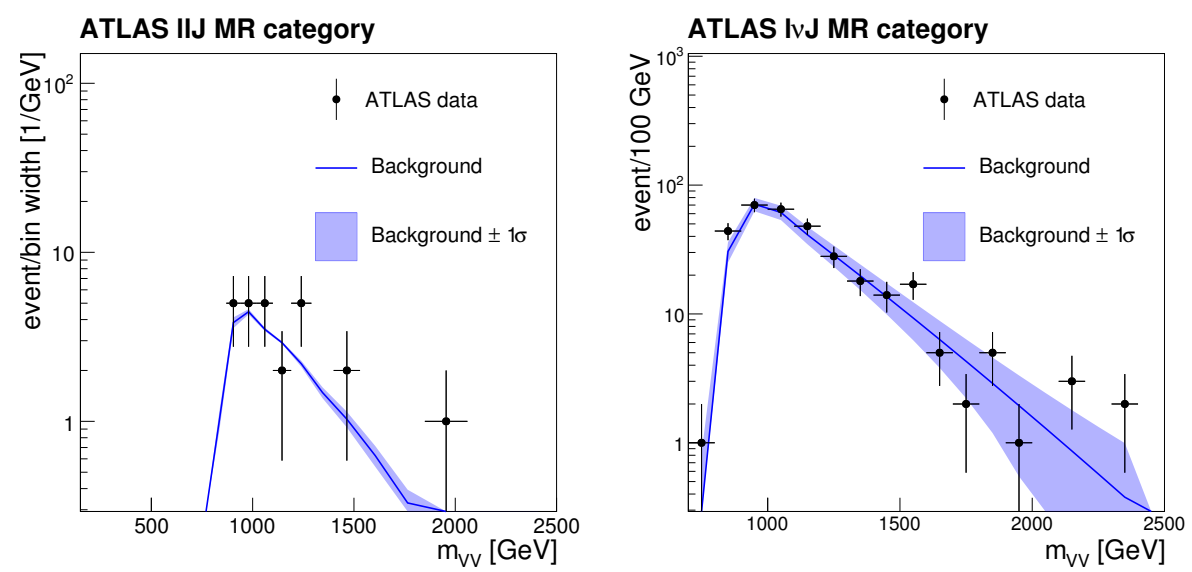

Figure 12. ATLAS ZV $\rightarrow \ell \ell \mathrm{J}$ (left) and $\mathrm{WV} \rightarrow \ell \nu \mathrm{J}$ (right) searches: comparison between the official ATLAS background (blue line) and its uncertainties (purple band) with the overlaid data of the $m_{\mathrm{JJ}}$ spectrum for the Merged Region (of the vector boson hadronic reconstruction) category.

\subsection{Emulation of ATLAS search}

\subsubsection{Description of the ATLAS analysis}

The ATLAS semileptonic search considers both the case in which the two quarks from the vector boson decay are reconstructed as a single merged jet (boosted regime), and the case in which they are reconstructed as two distinct jets (resolved regime). In this study, we focus on resonances heavier than $1.5 \mathrm{TeV}$, for which the merged regime largely drives the sensitivity. Thus we consider only the Merged Region (MR) categories of refs. [79, 80].

In both $\mathrm{ZV} \rightarrow \ell \ell \mathrm{J}$ and $\mathrm{WV} \rightarrow \ell \nu \mathrm{J}$ searches, the boosted jet is identified using the mass-drop filtering algorithm (as in the VV $\rightarrow$ JJ search). In addition, two same-flavour opposite-sign leptons, or one charged lepton and missing transverse energy (MET) are required. The events are selected online by single- or double-lepton based triggers. The detector coverage includes the tracker volume $(|\eta|<2.5)$ and the fiducial region of the electromagnetic calorimeter (for electrons) or the muon detector. The typical $p_{\mathrm{T}}$ threshold for the charged leptons and for MET is $25 \mathrm{GeV}$. The main backgrounds are inclusive $\mathrm{V}$ production (i.e. $\mathrm{Z}+$ jets for the $\ell \ell \mathrm{J}$ channel and $\mathrm{W}+$ jets for the $\ell \nu \mathrm{J}$ channel), as well as $t \bar{t}$ production.

\subsubsection{Statistical analysis}

We build the likelihood for the ATLAS semileptonic searches using the information documented in the HEPDATA database. The ATLAS collaboration estimates the background uncertainties separately for each lepton category. The electron $p_{\mathrm{T}}$ resolution is better than that of the muon in the high- $p_{\mathrm{T}}$ region. The systematic uncertainties associated with different background sources ( $t \bar{t}$ and electroweak components) are also treated separately. Nevertheless, the background distributions documented in the HEPDATA database (see figure 12) are presented jointly for electrons and muons. We model the signal distributions in the diboson mass spectrum with a Gaussian function, centred at the assumed resonance 
mass and with a width reflecting the experimental resolution. We assume a fixed value of $4 \%$ resolution in the $\ell \ell \mathrm{J}$ channel for all mass values. ${ }^{6}$ Similarly, we assume a fixed value of $10 \%$ resolution in the $\ell \nu \mathrm{J}$ channel for all mass values ${ }^{7}$ (see figure 1 in ref. [79]).

The signal distributions are normalised to the expected yield, as calculated from the theoretical cross section and the selection efficiency provided by the ATLAS collaboration.

We consider the following systematic uncertainties, treated as fully correlated across $m_{\mathrm{JJ}}$ histogram bins:

- Background uncertainty, provided by the ATLAS experiment (in HEPDATA).

- Signal normalisation uncertainty, which is separated into two further sub-categories: a common-across-channels systematic uncertainty corresponding to the luminosity measurement (2.8\%), and an additional term accounting for all types of scale and efficiency systematic effects $(10 \%)$. The latter is treated as uncorrelated between the $\ell \ell \mathrm{J}$ and $\ell \nu \mathrm{J}$ channels.

Given the approximations that we have introduced to model the signal, we do not expect our statistical analysis to produce results matching with high accuracy the public ATLAS results. Similarly to the procedure followed for the emulation of the fully hadronic ATLAS search, we introduce a fudge factor to reduce this discrepancy. The value of the fudge factor is chosen such that the expected exclusion limits produced by this study agree with the official limits by ATLAS. It is found to be between 0.8 and 1.2 in the resonance mass range of interest, slowly decreasing for larger mass values (figure 13). With this correction, our calculated exclusion limits are in good agreement with the public ATLAS results (figure 14).

\subsection{Emulation of CMS search}

\subsubsection{Description of the CMS analysis}

The CMS semileptonic analyses [21] are performed with data collected by single-lepton triggers for the $\ell \nu \mathrm{J}$ channel and double-lepton triggers for the $\ell \ell \mathrm{J}$ channel. Jets are identified as boosted vector bosons using the same algorithm employed for the fully hadronic search (see section 3). Similarly to the strategy developed in the fully hadronic search, LP and HP categories are introduced, based on the value of $\tau_{21}$, to increase the analysis sensitivity.

The analysis is performed by using a $\mathrm{G}_{\text {bulk }}$ graviton as the benchmark signal model. In order to facilitate the interpretation of the search results in other theoretical models, the CMS collaboration provides the reconstruction efficiencies of leptonic and hadronic $\mathrm{W}_{L}$

\footnotetext{
${ }^{6}$ The signal resolution for a $m_{\mathrm{X}}=2 \mathrm{TeV}$ resonance in the $\ell \ell \mathrm{J}$ channel is $4 \%$, decreasing to $3 \%$ for lower masses [80]. We assume a fixed resolution to simplify the analysis.

${ }^{7}$ In the case of the $\ell \nu \mathrm{J}$ channel, the reconstruction of the resonance mass requires an assumption on the longitudinal momentum of the outgoing neutrino that is not detected. In practice, this is estimated from the MET measurement combined with a W mass constraint. The diboson resonance mass is subsequently computed using the jet, lepton and calculated neutrino momenta. The mass resolution in this channel is degraded compared to the $\ell \ell J$ channel.
} 


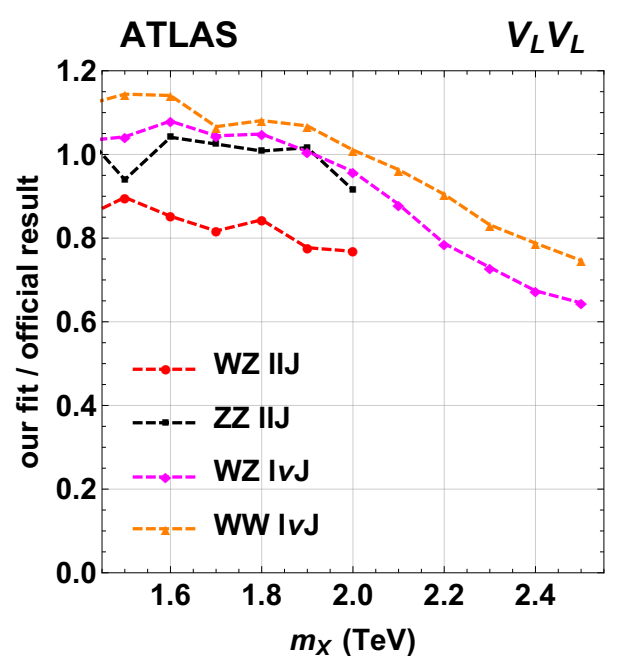

Figure 13. ATLAS semileptonic searches: fudge factor as a function of the mass $m_{X}$ of the exotic resonance, calculated via the ratio of observed exclusion limits obtained with this study to the ones of the official ATLAS result, for the $\mathrm{W}^{\prime} \rightarrow \mathrm{W}_{L} \mathrm{Z}_{L}$ (red) and $\mathrm{G}_{\text {bulk }} \rightarrow \mathrm{Z}_{L} \mathrm{Z}_{L}$ (black) signal hypotheses in the $\ell \ell J$ channel, and for the $\mathrm{W}^{\prime} \rightarrow \mathrm{W}_{L} \mathrm{Z}_{L}$ (magenta) and $\mathrm{G}_{\mathrm{bulk}} \rightarrow \mathrm{W}_{L} \mathrm{~W}_{L}$ (orange) signal hypotheses in the $\ell \nu \mathrm{J}$ channel.

and $\mathrm{Z}_{L}$ in the $\mathrm{HP}$ category, as function of the boson's $p_{\mathrm{T}}$ and $\eta$. Those $2 \mathrm{D}$ efficiency maps include the effects of the pruned jet mass and $\tau_{21}$ selections, as well as the resonance mass reconstruction.

\subsubsection{Statistical analysis}

The background model is extracted by fitting the $m_{\mathrm{VV}}$ data distributions for each lepton flavour with a levelled exponential

$$
f\left(m_{\mathrm{VV}}\right)=N \exp \left[-\frac{m_{\mathrm{VV}}}{\sigma+k \cdot m_{\mathrm{VV}}}\right]
$$

where $N, k$ and $\sigma$ are free parameters. This function saturates in the high $m_{\mathrm{VV}}$ region, and is meant to describe events where $m_{\mathrm{VV}}$ was significantly mismeasured. For example, this may happen if a high $p_{T}$ muon leaves a nearly straight track barely bent by the magnetic field, or if the calculation of the neutrino momentum fails. In practice, this function is used in ref. [21] to model the HP category with $k$ as a free parameter, whereas for the LP category $k$ can be set to 0 . In the $\ell \ell \mathrm{J}$ channel we focus on the $m_{\mathrm{VV}}>700 \mathrm{GeV}$ region, and we merge the contents of the (publicly available) $50 \mathrm{GeV}$ wide bins to obtain a uniform, $100-\mathrm{GeV}$-wide binning for the $m_{\mathrm{VV}}$ distribution. We use the diagonalised uncertainties from the fit $\left(\sigma_{\lambda_{i}}\right.$, with $\left.i=0,1,2\right)$ as background uncertainties. Figures 15 and 16 show the comparison between the fits produced in this study and the official CMS fits on the data distributions.

We model the signal distributions in the diboson mass spectrum with a Gaussian function. The HP signal yield is calculated from the theoretical cross section and the selection efficiency obtained from the algorithm described in ref. [21]. The first step in this 

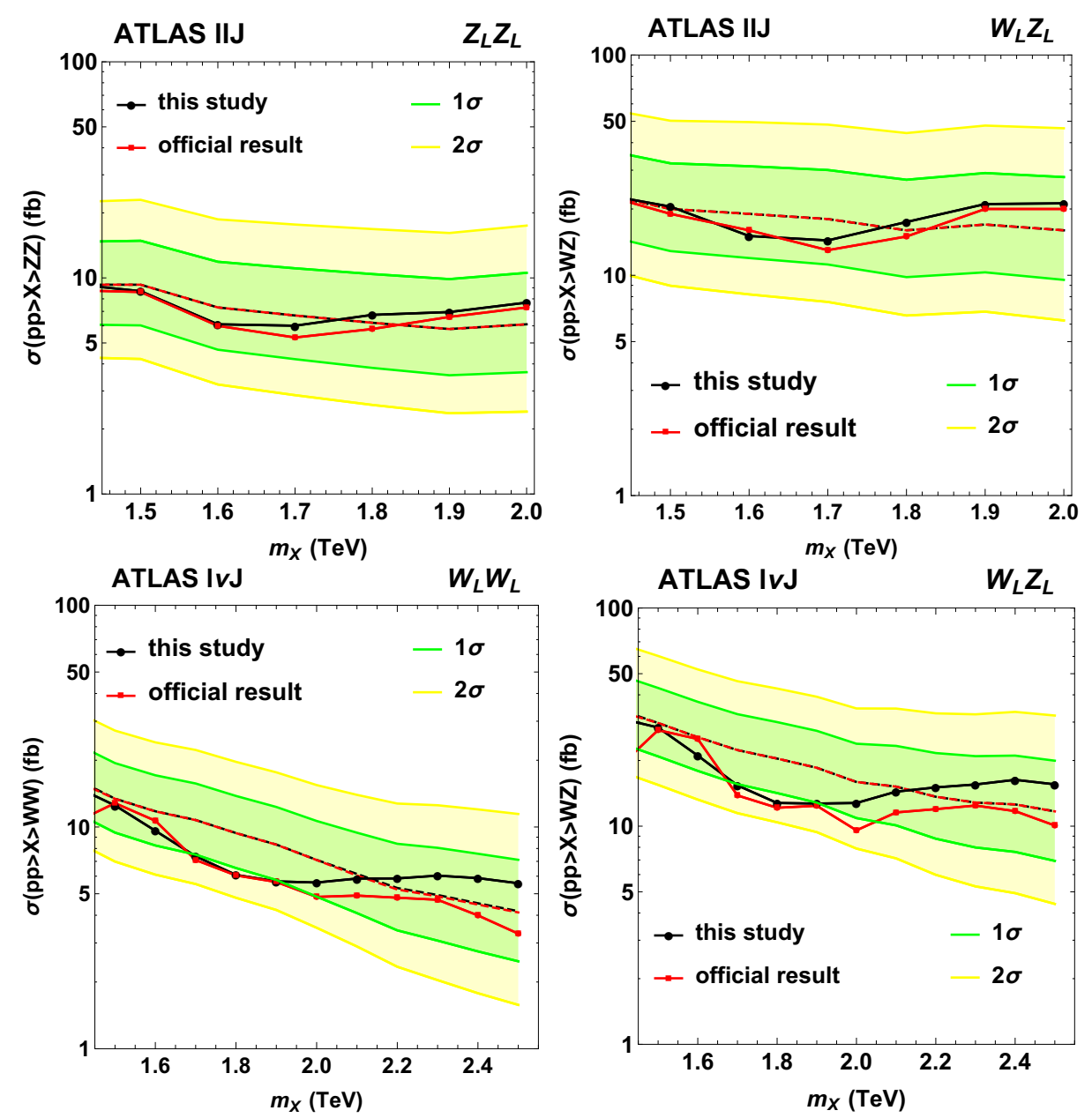

Figure 14. ATLAS semileptonic searches: expected (dashed lines) and observed (continuous lines) exclusion limits on exotic production cross sections as a function of the resonance mass $m_{X}$ obtained with this study (black), and comparison with the official CMS results (red) for $\mathrm{G}_{\text {bulk }} \rightarrow \mathrm{Z}_{L} \mathrm{Z}_{L}$ (top left), $\mathrm{W}^{\prime} \rightarrow \mathrm{W}_{L} \mathrm{Z}_{L}$ (top right), $\mathrm{G}_{\text {bulk }} \rightarrow \mathrm{W}_{L} \mathrm{~W}_{L}$ (bottom left) and $\mathrm{W}^{\prime} \rightarrow \mathrm{W}_{L} \mathrm{Z}_{L}$ (bottom right) signal hypotheses in the $\ell \ell \mathrm{J}$ (top) and $\ell \nu \mathrm{J}$ (bottom) channels. The green and yellow bands represent the one and two sigma variations around the median expected limits calculated in this study, with all the corrections described in the text included.

process is the generation of signal samples with the Madgraph5 generator as described in section 2. We then apply acceptance selections on the leptons and generator-level jets, and use the 2D efficiency maps to emulate the V-boson reconstruction and tagging processes. Finally, we apply a $90 \%$ correction to account for $b$-jet veto inefficiencies. Considering the approximations made, this procedure is expected to reproduce the official CMS results within a $10 \%$ accuracy. The HP-category efficiencies that we obtain are consistent with the nominal $\mathrm{G}_{\text {bulk }} \rightarrow \mathrm{W}_{L} \mathrm{~W}_{L}$ efficiencies for $m_{\mathrm{X}}=1.2 \mathrm{TeV}$ within $6 \%$.

The LP category signal efficiencies are generally not provided, but examples of the $\mathrm{LP} / \mathrm{HP}$ efficiency ratios are given for a $\mathrm{G}_{\text {bulk }}$ signal with $m_{\mathrm{X}}=1.2 \mathrm{TeV}$. The ratio is 0.47 (0.25) for the $\ell \ell \mathrm{J}(\ell \nu \mathrm{J})$ channel. The reason for the efficiency difference between the two 

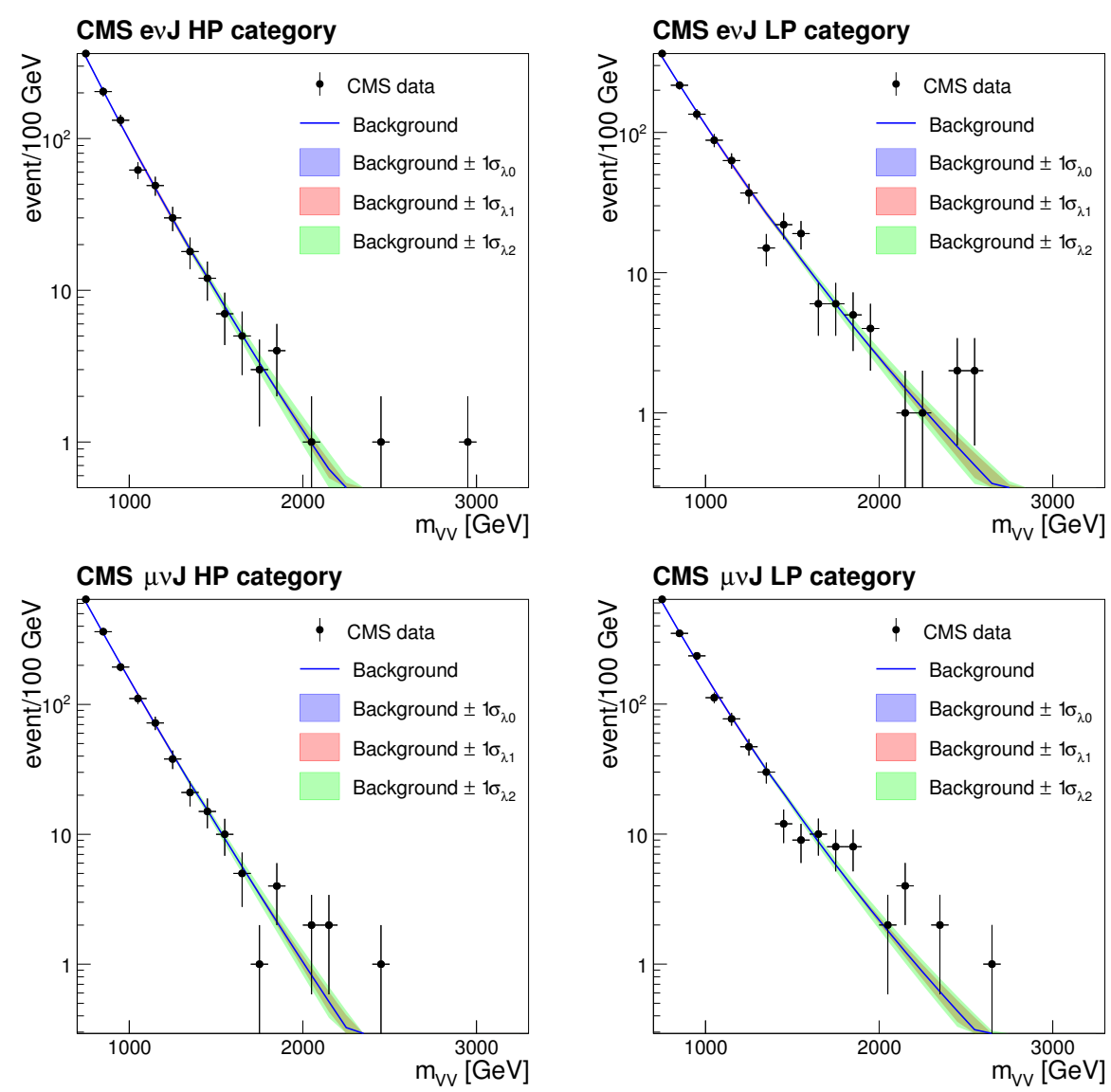

Figure 15. CMS WV $\rightarrow \ell \nu \mathrm{J}$ search: comparison between the official CMS background (blue line) and the background modelling with uncertainties employed by this study (coloured bands), with the overlaid data of the $m_{\mathrm{JJ}}$ spectrum for the HP (left-hand side) and LP (right-hand side) categories, plotted separately for the electron (top) and the muon (bottom) channels.

cases lies in the different boosted jet selection applied in the two channels. We make the assumption that we can use the same LP/HP ratio for all mass points under consideration in this study, and use the values above to estimate the expected signal yields in the LP category. Finally, the $\tau_{21}$ categorisation is not sensitive to the nature of the resonance, ${ }^{8}$ therefore we use the same LP/HP ratio also for the $\mathrm{W}^{\prime}$ signal hypothesis.

We consider the following systematic uncertainties, treated as fully correlated across $m_{\mathrm{JJ}}$ histogram bins:

- Background uncertainty, extracted from our fit to the data distributions.

- Signal normalisation uncertainty, which is separated into two further sub-categories: a common-across-channels systematic uncertainty corresponding to the luminosity measurement $(2.2 \%)$, and an additional uncertainty covering all lepton-related uncertainties (3.7\% for electrons, $3 \%$ for muons), applied separately for the $\ell \ell \mathrm{J}$ and $\ell \nu \mathrm{J}$ channels.

\footnotetext{
${ }^{8}$ Provided that the polarisation of the final state bosons is the same for both models.
} 

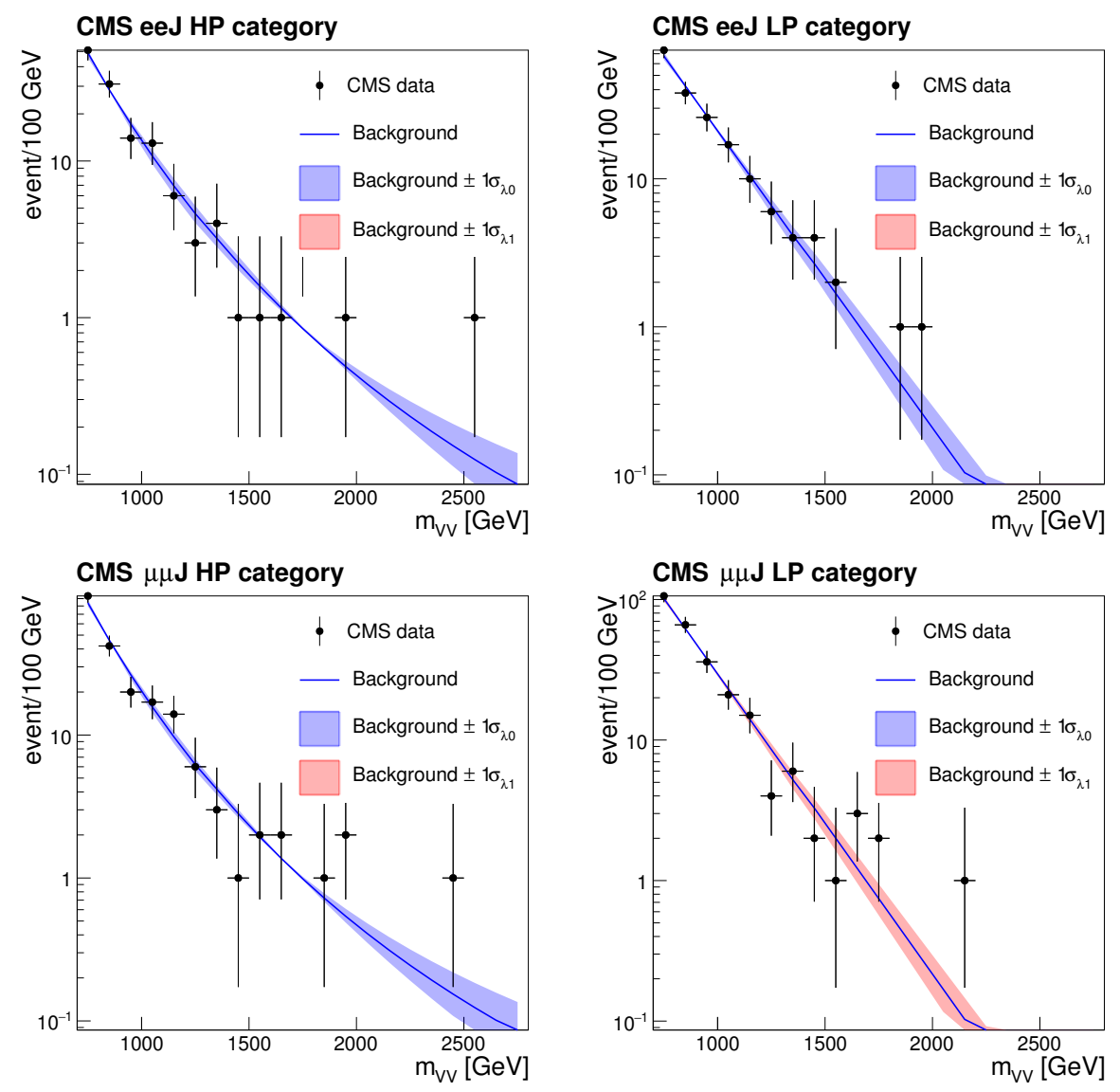

Figure 16. CMS ZV $\rightarrow \ell \ell J$ search: comparison between the official CMS background (blue line) and the background modelling with uncertainties employed by this study (coloured bands), with the overlaid data of the $m_{\mathrm{JJ}}$ spectrum for the HP (left-hand side) and LP (right-hand side) categories, plotted separately for the electron (top) and the muon (bottom) channels.

- Signal purity category migration uncertainty, which covers the effects of events "migrating" from the HP to the LP category, or vice-versa. This uncertainty amounts to $9 \%$ and $24 \%$, respectively.

As already discussed in previous sections, we apply a fudge factor to account for differences between our background description and the one from the public CMS result, as well as for the approximations introduced in the signal modelling (figure 17). With this correction, our calculated exclusion limits are in good agreement with the public CMS results (figure 18). The statistical uncertainties (one- and two-sigma coverage bands) are $\approx 50 \%$ smaller than expected, as they have been calculated with the asymptotic CLs method, which is known to underestimate uncertainties in tests with small statistics.

We use the same procedure to recast the results in the context of a $\mathrm{W}^{\prime} \rightarrow \mathrm{W}_{L} \mathrm{Z}_{L}$ signal search, with the results presented in figure 19. The jet mass selection for the $\ell \ell \mathrm{J}$ channel is $70<m_{J}<110 \mathrm{GeV}$, to be compared with $65<m_{J}<105 \mathrm{GeV}$ for the $\ell \nu \mathrm{J}$ analysis. This 


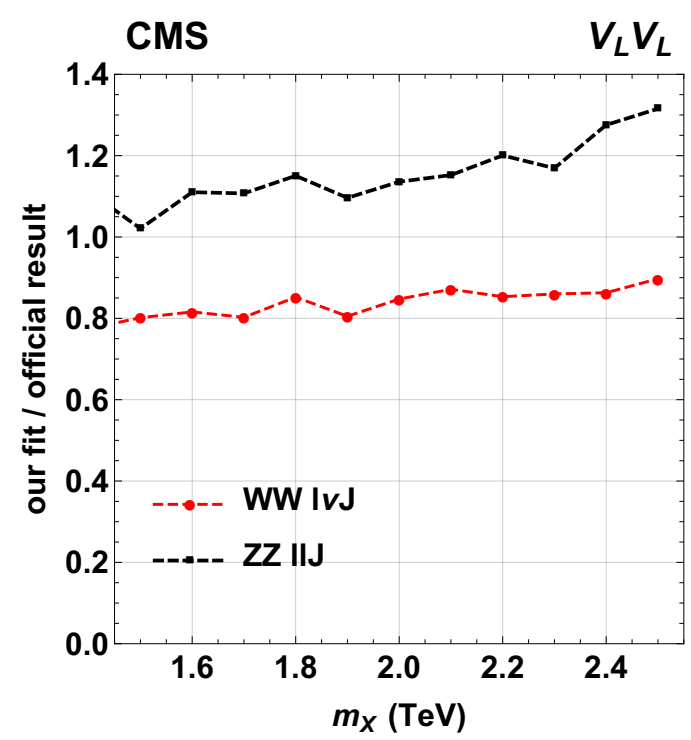

Figure 17. CMS semileptonic searches: fudge factor as a function of the mass $m_{X}$ of the exotic resonance, calculated via the ratio of observed exclusion limits obtained with this study to the ones of the official CMS result for the $\mathrm{G}_{\mathrm{bulk}} \rightarrow \mathrm{W}_{L} \mathrm{~W}_{L}$ (red) and $\mathrm{G}_{\mathrm{bulk}} \rightarrow \mathrm{Z}_{L} \mathrm{Z}_{L}$ (black) semileptonic analyses.
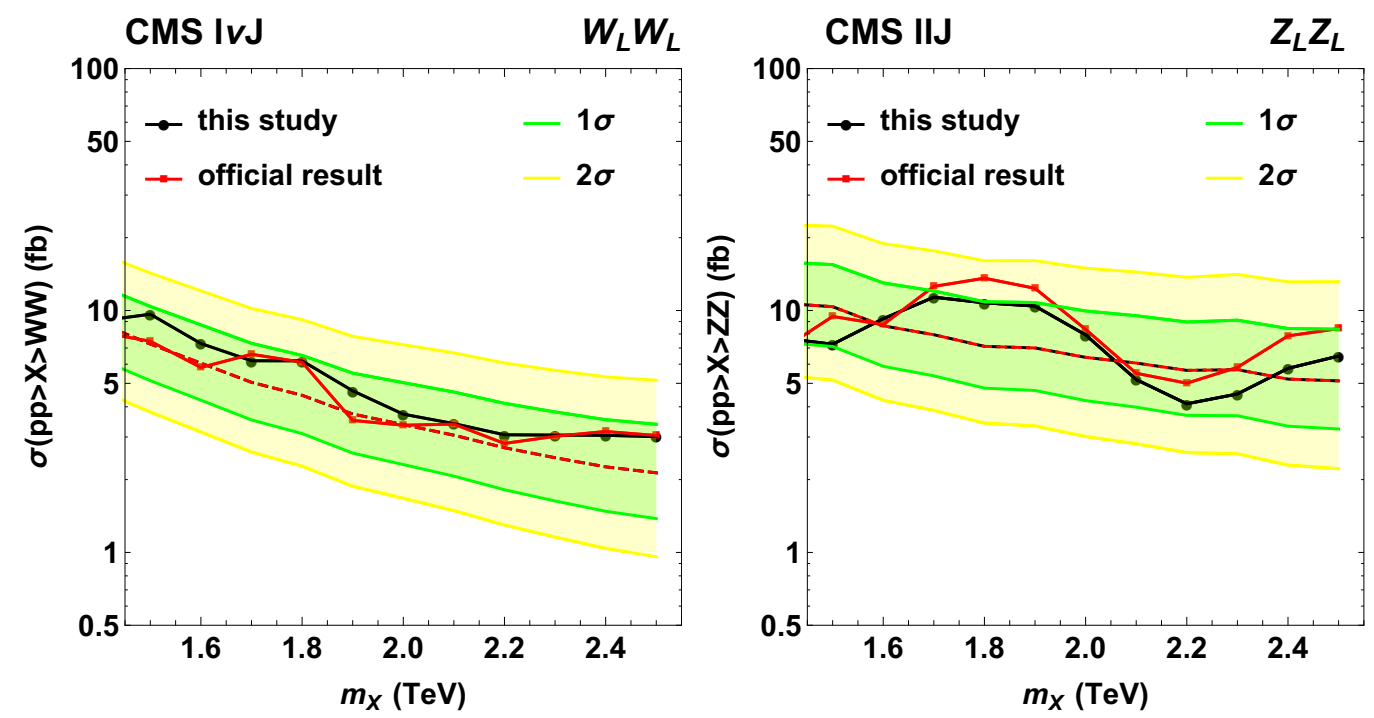

Figure 18. CMS semileptonic searches: expected (dashed lines) and observed (continuous lines) exclusion limits on exotic production cross sections as a function of the resonance mass $m_{X}$ obtained with this study (black), and comparison with the official CMS results (red) for the $\mathrm{G}_{\text {bulk }} \rightarrow \mathrm{W}_{L} \mathrm{~W}_{L}$ search in the $\ell \nu \mathrm{J}$ channel (left) and the $\mathrm{G}_{\text {bulk }} \rightarrow \mathrm{Z}_{L} \mathrm{Z}_{L}$ search in the $\ell \ell \mathrm{J}$ channel (right). The green and yellow bands represent the one and two sigma variations around the median expected limits calculated in this study, with all the corrections described in the text included.

choice was made in order to optimise the search for a neutral resonance (at the expense of the search for a charged one). Since the $\ell \nu \mathrm{J}$ channel mass window is shifted to a region with more background, the signal sensitivity for the $\ell \nu \mathrm{J}$ channel is reduced. 

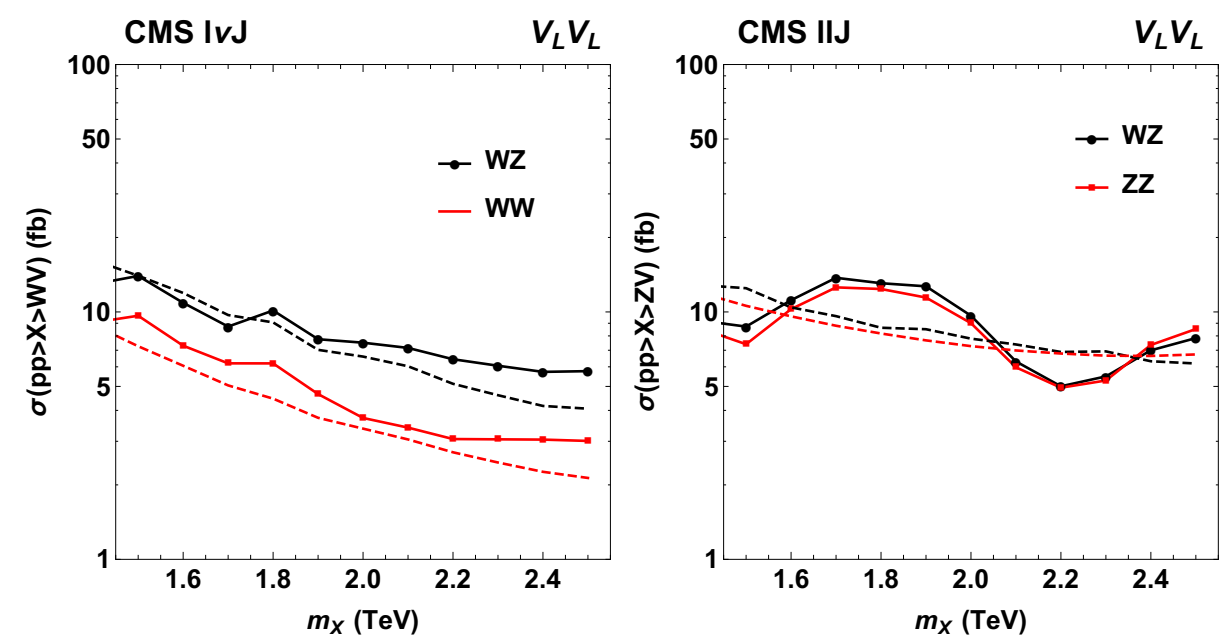

Figure 19. CMS semileptonic searches: expected (dashed lines) and observed (continuous lines) exclusion limits on exotic production cross section as a function of the resonance mass $m_{X}$ obtained with this study for the $\mathrm{G}_{\text {bulk }} \rightarrow \mathrm{W}_{L} \mathrm{~W}_{L}$ (red) and $\mathrm{W}^{\prime}$ (black) signal hypotheses in the $\ell \nu \mathrm{J}$ channel (left) and for the $\mathrm{G}_{\text {bulk }} \rightarrow \mathrm{Z}_{L} \mathrm{Z}_{L}$ (red) and $\mathrm{W}^{\prime}$ (black) signal hypotheses in the $\ell \ell \mathrm{J}$ channel (right).

\subsection{Combined LHC results of semi-leptonic searches}

Here we discuss the combination of the ATLAS and CMS searches in the semileptonic channels $(\ell \nu \mathrm{J}$ and $\ell \ell \mathrm{J})$ and the interpretation of the results under different signal hypotheses, with final states including a leptonic $\mathrm{W}(\rightarrow \ell \nu)$ or $\mathrm{Z}(\rightarrow \ell \ell)$ decay. The results are summarised in figure 20.

Under the hypothesis of a $\mathrm{Z}_{\mathrm{L}} \mathrm{Z}_{\mathrm{L}}$ benchmark model, only the $\ell \ell \mathrm{J}$ searches are relevant. In this channel, CMS observes a small excess $(\approx 1 \sigma)$ between 1.7 and $1.9 \mathrm{TeV}$, while ATLAS $\mathrm{a}<1 \sigma$ excess between 1.9 and $2.0 \mathrm{TeV}$, driven by the presence of one event in the highest bin of the merged analysis distribution. The combination of the two channels results in a more stringent limit and a moderate excess of the order of $1 \sigma$ around $1.9 \mathrm{TeV}$. Above 2 $\mathrm{TeV}$, ATLAS has not published their search results and the limit considered here is the one provided by CMS. While the significance of the observed deviation is too small to cause any excitement, the sensitivity of this analysis is strongly reduced. This has implications for the combination result discussed in section 5 .

On the contrary, under the hypothesis of a $\mathrm{W}_{\mathrm{L}} \mathrm{W}_{\mathrm{L}}$ benchmark model, only the $\ell \nu \mathrm{J}$ searches are relevant. An observed upward fluctuation around $m_{\mathrm{VV}}=1.8 \mathrm{TeV}$ in the CMS data spectrum is compensated by a downward fluctuation in the same region for the ATLAS data. The two deviations effectively cancel each other, resulting into observed exclusion limits which are consistent with the experimental sensitivity and the backgroundonly hypothesis expectations.

For the $\mathrm{W}_{\mathrm{L}} \mathrm{Z}_{\mathrm{L}}$ benchmark model, we are able to combine the experimental results in the $\ell \ell \mathrm{J}$ and $\ell \nu \mathrm{J}$ channels. The sensitivity and the relative weight of the $\ell \ell \mathrm{J}$ channel is larger than those of the $\ell \nu \mathrm{J}$ channel in the combination. Similar to the interpretation of the search results in the $\mathrm{Z}_{\mathrm{L}} \mathrm{Z}_{\mathrm{L}}$ signal hypothesis, we observe here that the combined results give a small excess $(\approx 1 \sigma)$ around $m_{\mathrm{VV}}=1.9 \mathrm{TeV}$. 

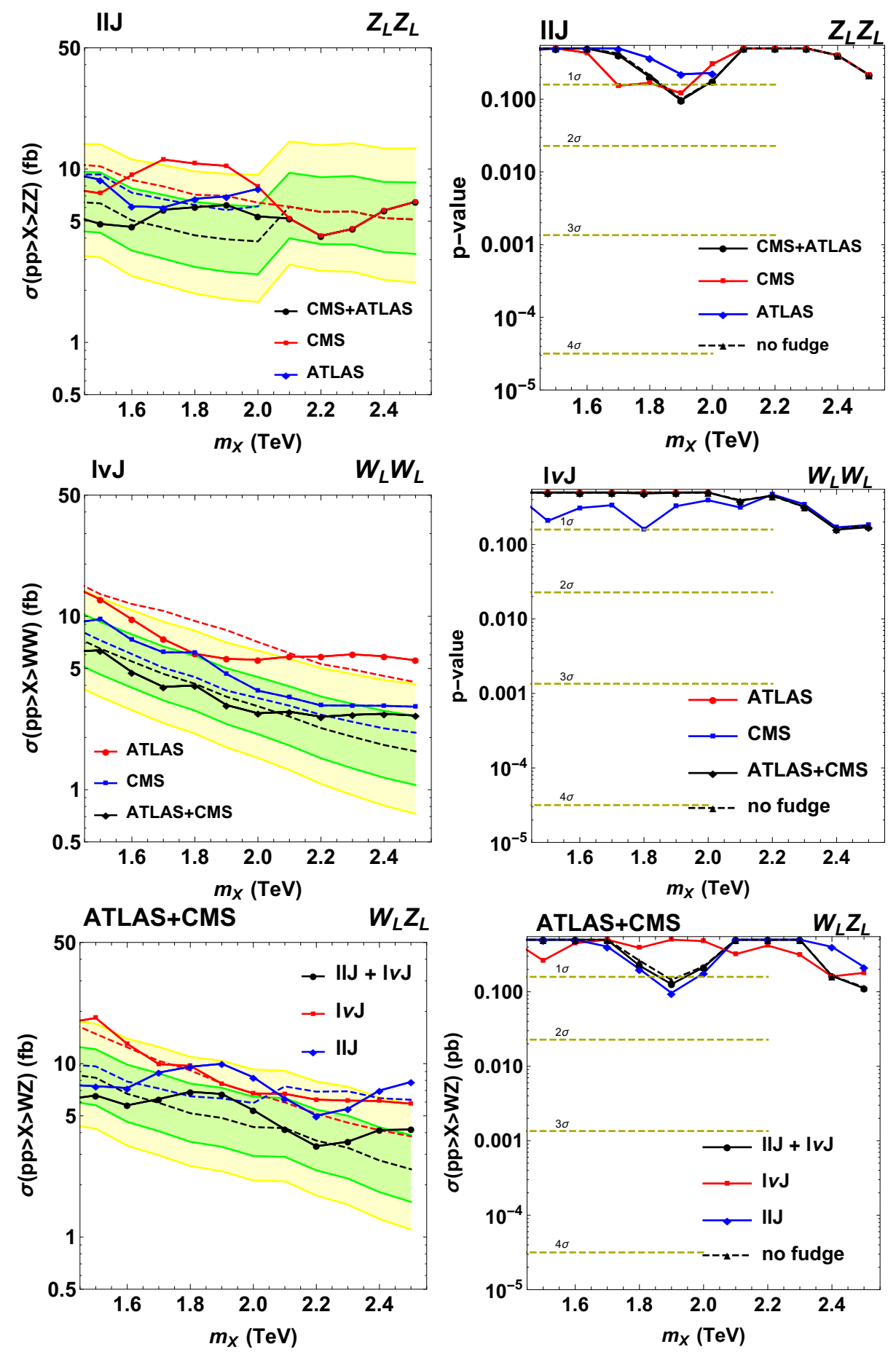

Figure 20. Combination of semileptonic searches for $\mathrm{G}_{\text {bulk }} \rightarrow \mathrm{Z}_{L} \mathrm{Z}_{L}$ (top), $\mathrm{G}_{\text {bulk }} \rightarrow \mathrm{W}_{L} \mathrm{~W}_{L}$ (middle) and $\mathrm{W}^{\prime} \rightarrow \mathrm{W}_{L} \mathrm{Z}_{L}$ (bottom) selections and signal hypotheses, and as a function of the resonance mass $m_{\mathrm{X}}$ obtained with the emulation of the ATLAS (red) and CMS (blue) searches and their combination (black). Left: expected (dashed lines) and observed (continuous lines) exclusion limits on exotic production cross section. The green and yellow bands represent the one and two sigma variations around the median expected limits. The results include the correction factors discussed in the text. Right: likelihood ratio $p$-values. The dashed black curve corresponds to the combined search without the corrections discussed in the text. 


\section{Combination of hadronic and semi-leptonic channels}

This section is dedicated to the combination of both hadronic and semileptonic channels by ATLAS and CMS under different signal hypotheses. The searches in the JJ and $\ell \nu \mathrm{J}$ channels contribute to constrain a hypothetical $\mathrm{G}_{\mathrm{bulk}} \rightarrow \mathrm{W}_{L} \mathrm{~W}_{L}$ production; searches in the JJ and $\ell \ell J$ channels enter the combination for the interpretation of the results in a $\mathrm{G}_{\text {bulk }} \rightarrow \mathrm{Z}_{L} \mathrm{Z}_{L}$ signal scenario. Finally, all six searches (i.e. results in three channels by two experiments) enter the combination in the $\mathrm{W}^{\prime} \rightarrow \mathrm{W}_{L} \mathrm{Z}_{L}$ signal hypothesis.

The exclusion limits on production cross section, likelihood-ratio $p$-values, and best-fit cross sections as a function of a hypothetical resonance mass are summarised in figure 21 . Scans of the profile likelihood as a function of the exotic production cross section for $m_{X}$ $=1.9$ and $2.0 \mathrm{TeV}$ (mass values of largest excesses for the benchmark models considered) are given in figure 22. The sensitivity of the search in the $\mathrm{G}_{\mathrm{bulk}} \rightarrow \mathrm{Z}_{L} \mathrm{Z}_{L}$ signal hypothesis is dominated by the semileptonic analyses below $1.9-2.0 \mathrm{TeV}$ and the fully hadronic searches at higher mass ranges. The largest deviation is observed at $m_{X}=1.9 \mathrm{TeV}$, driven by the ATLAS excess in the VV $\rightarrow$ JJ channel. The overall significance remains above $3 \sigma$. The preferred cross section for a hypothetical $\mathrm{G}_{\text {bulk }} \rightarrow \mathrm{Z}_{L} \mathrm{Z}_{L}$ signal as calculated in the $\ell \ell \mathrm{J}$ channel is $\approx 2 \mathrm{fb}$ and increases to $\approx 9 \mathrm{fb}$ for the $\mathrm{JJ}$ channel. When combined, the estimated cross section is $5 \mathrm{fb}$. The combination of the two channels reduces the exotic cross section favoured by the JJ results, and alleviates the potential disagreement between different channels, without reducing the overall significance of the excess. In other words, the combination of the two channels leads to a more coherent picture of the results by the two experiments. This is also evident from the profile likelihood scans shown in figure 21: given the uncertainty on the best-fit exotic production cross section, and contrary to what one might expect by considering the individual exclusion limits, the results obtained in different final states are not in tension with each other. In addition, the combination pushes the excess to mass values below $2 \mathrm{TeV}$.

The picture is quite different in the $\mathrm{G}_{\mathrm{bulk}} \rightarrow \mathrm{W}_{L} \mathrm{~W}_{L}$ signal interpretation. The lack of a significant excess in the $\ell \nu \mathrm{J}$ channels is strong enough to reduce the significance of the JJ excess below the $1 \sigma$ threshold. The combination of the ATLAS and CMS results disfavours the hypothesis of a resonance decaying exclusively to WW (an interpretation which in any case would be difficult to justify phenomenologically).

Finally, the interpretation of the results in the context of a $\mathrm{W}^{\prime}$ signal hypothesis lies between the $\mathrm{G}_{\text {bulk }} \rightarrow \mathrm{Z}_{L} \mathrm{Z}_{L}$ and $\mathrm{G}_{\text {bulk }} \rightarrow \mathrm{W}_{L} \mathrm{~W}_{L}$ scenarios: the $\ell \nu \mathrm{J}$ analyses are more sensitive than the fully hadronic ones, but their contribution is not as dominant as in the $\mathrm{G}_{\text {bulk }} \rightarrow \mathrm{W}_{L} \mathrm{~W}_{L}$ case. Nevertheless, the excess survives above the $3 \sigma$ threshold, thanks to the presence of a moderate excess in the $\ell \ell \mathrm{J}$ search around the same mass region. Overall, the estimated cross section of a hypothetical exotic signal is strongly reduced: the bestfit value changes from $\approx 10 \mathrm{fb}$ (when using the $\mathrm{JJ}$ channel results only) to $\approx 5 \mathrm{fb}$ (when combining the $\mathrm{JJ}, \ell \nu \mathrm{J}$ and $\ell \ell \mathrm{J}$ channels). At this smaller cross section value, the outcome of the searches in the different channels is quite coherent, as shown in the profile likelihood scans depicted in figure 21. The mitigating effect of the $\ell \ell \mathrm{J}$ result is evident if one compares the $\ell \nu \mathrm{J}$-and- $\ell \ell \mathrm{J}$ combined likelihood scan for the $\mathrm{W}^{\prime}$ combination to the likelihood scans 
in the semileptonic searches. The $\mathrm{W}_{\mathrm{L}} \mathrm{Z}_{\mathrm{L}}$ curve is much more similar to the $\mathrm{Z}_{\mathrm{L}} \mathrm{Z}_{\mathrm{L}}$ curve in the $\ell \ell \mathrm{J}$ channel than to the $\mathrm{W}_{\mathrm{L}} \mathrm{W}_{\mathrm{L}}$ curve in the $\ell \nu \mathrm{J}$ channel.

In conclusion, a resonance with a production cross section of $\sim 5 \mathrm{fb}$ and mass between 1.9 and $2.0 \mathrm{TeV}$ is the scenario most consistent with the experimental results out of all benchmark models considered in this study, as long as it does not decay exclusively to a $\mathrm{W}_{\mathrm{L}} \mathrm{W}_{\mathrm{L}}$ final state.

An example of the model independent combination of the $\mathrm{Z}_{\mathrm{L}} \mathrm{Z}_{\mathrm{L}}$ and $\mathrm{W}_{\mathrm{L}} \mathrm{W}_{\mathrm{L}}$ channels is shown in figure 23 . In this case, one considers a resonance that can decay to both $\mathrm{W}_{\mathrm{L}} \mathrm{W}_{\mathrm{L}}$ and $\mathrm{Z}_{\mathrm{L}} \mathrm{Z}_{\mathrm{L}}$, with the relative branching fraction determined by the $r$ parameter introduced in eq. (2.1). For $r \rightarrow 0$ one recovers the $\mathrm{G}_{\text {bulk }} \rightarrow \mathrm{Z}_{L} \mathrm{Z}_{L}$ case, while for $r \rightarrow \infty$ one recovers the $\mathrm{G}_{\text {bulk }} \rightarrow \mathrm{W}_{L} \mathrm{~W}_{L}$ limit. It should be noted that for this combination we use a common mass window for the ATLAS analyses, namely the one that corresponds to the ZZ search, giving the best overall sensitivity (see section 3). Therefore, the results obtained here on the $\mathrm{W}_{\mathrm{L}} \mathrm{W}_{\mathrm{L}}$ exclusion limits and $p$-values are somewhat different than the ones presented in figure 21 .

The results obtained for generic values of $r$ are similar to the $\mathrm{Z}_{\mathrm{L}} \mathrm{Z}_{\mathrm{L}}$ case, i.e. they point to an overall excess. The size of the excess is reduced to $2 \sigma$, with a best-fit exotic production cross section around $4 \mathrm{fb}$. Particularly interesting is the $r=2$ case, corresponding to a resonance with universal couplings to the pseudo-Goldstone bosons. In this case, despite the fact that $\mathcal{B}\left(X \rightarrow \mathrm{W}_{\mathrm{L}} \mathrm{W}_{\mathrm{L}}\right)=2 \mathcal{B}\left(X \rightarrow \mathrm{Z}_{\mathrm{L}} \mathrm{Z}_{\mathrm{L}}\right)$, the combined deviation is found to have $\mathrm{a} \approx 2.4 \sigma$ significance for a cross section of $\approx 4 \mathrm{fb}$.

It may be interesting to comment here on how the statistical methods that we have employed in this study compare with the simplified practices used by the theoretical community. A standard technique employed in many theoretical papers is to assume Gaussian likelihoods for the cross section of hypothetical signals, taking as central value the difference between the observed and expected limits, and as standard deviation the expected (95\% C.L.) limit divided by 1.96. Then, one can use the cross sections and uncertainties as estimated in the various channels, and calculate a weighted average. This method should, in principle, work well for cases in which the fitted cross section comes with a relatively small uncertainty (which is, typically, not the case in most searches) and the systematic uncertainties can be considered as uncorrelated among channels and experiments (which may, or may not be the case). As an example, we note that the simplified combination of the search results in the $\mathrm{G}_{\text {bulk }} \rightarrow \mathrm{W}_{L} \mathrm{~W}_{L}$ interpretation yields a best-fit cross section of $2.5 \pm 1.6 \mathrm{fb}(2.5 \pm 1.4 \mathrm{fb})$ at $m_{\mathrm{X}}=1.9 \mathrm{TeV}(2.0 \mathrm{TeV})$, to be compared with our result of $0.75_{-0.75}^{+1.67} \mathrm{fb}\left(1.1_{-1.1}^{+1.4} \mathrm{fb}\right)$. Similarly, the simplified combination in the $\mathrm{G}_{\text {bulk }} \rightarrow \mathrm{Z}_{L} \mathrm{Z}_{L}$ interpretation yields a best-fit cross section of $4.7 \pm 1.9 \mathrm{fb}(4.4 \pm 1.8 \mathrm{fb})$ at $m_{\mathrm{X}}=1.9 \mathrm{TeV}$ $(2.0 \mathrm{TeV})$, to be compared with our result of $5.2_{-1.6}^{+2.1} \mathrm{fb}\left(4.2_{-1.2}^{+1.9} \mathrm{fb}\right)$.

While more data is needed to clarify the situation, the results from the analysis of the diboson searches is unquestionably one of the most interesting outcomes of the ATLAS and CMS exotic programmes during the first LHC run. The situation is even more intriguing if one adds to the picture the $\approx 2 \sigma$ excess at $1.8-1.9 \mathrm{TeV}$ observed by CMS in a $\mathrm{W} H$ resonance search. The $\mathrm{W}^{\prime}$ results shown in figure 21 emerge as the most promising hint in the quest for a new heavy resonance in the ATLAS and CMS data, as already pointed out in ref. [39]. 

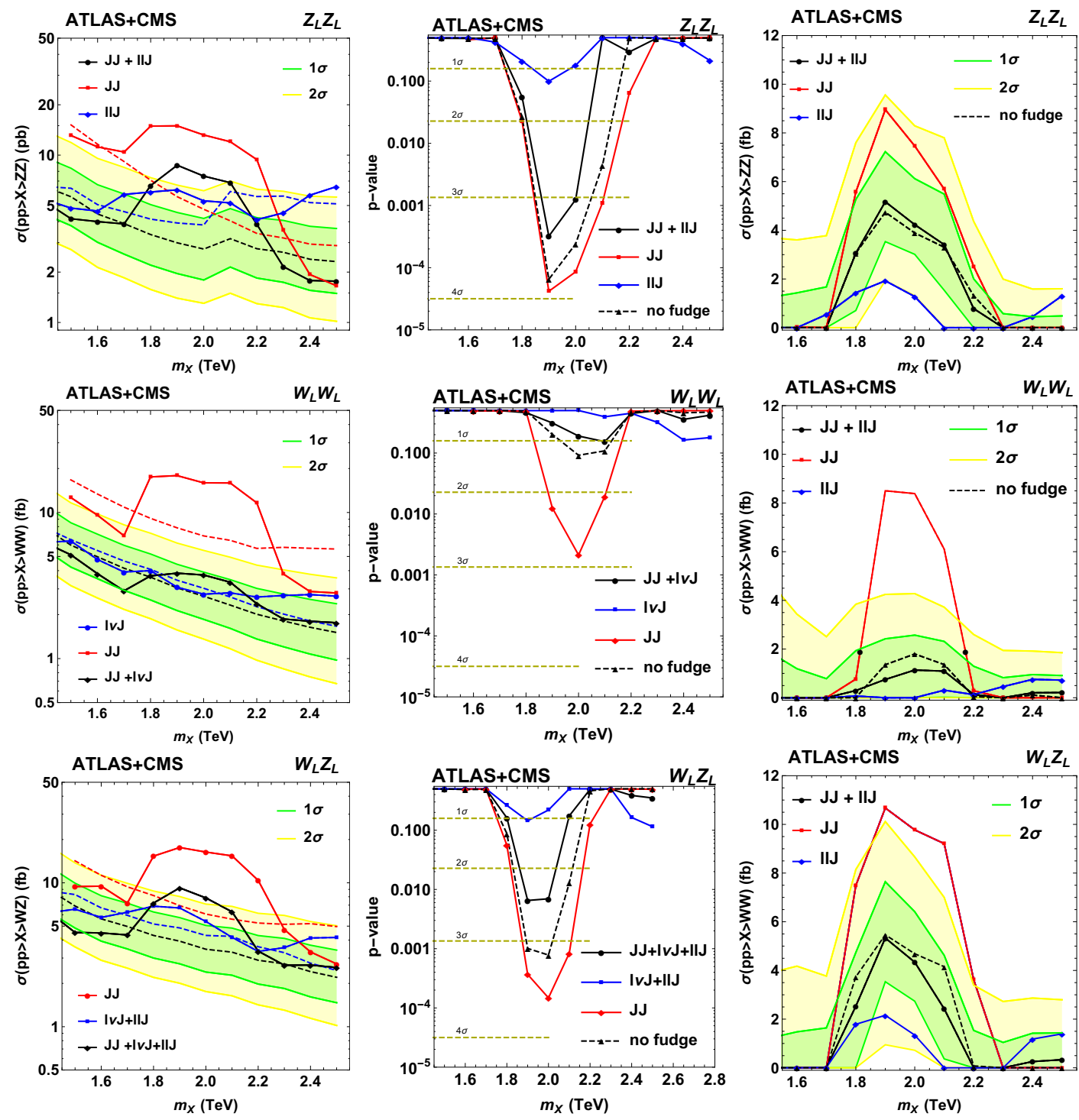

Figure 21. Combination of all ATLAS and CMS resonance searches for $\mathrm{G}_{\mathrm{bulk}} \rightarrow \mathrm{Z}_{L} \mathrm{Z}_{L}$ (top), $\mathrm{G}_{\text {bulk }} \rightarrow \mathrm{W}_{L} \mathrm{~W}_{L}$ (middle) and $\mathrm{W}^{\prime} \rightarrow \mathrm{W}_{L} \mathrm{Z}_{L}$ (bottom) selections and signal hypotheses, and as a function of the resonance mass $m_{\mathrm{X}}$ carried out in the hadronic (red) and semileptonic (blue) channels and their combination (black). The results include all correction factors discussed in the text. Left: expected (dashed lines) and observed (continuous lines) exclusion limits on exotic production cross section. The green and yellow bands represent the one and two sigma variations around the median expected limits. Middle: likelihood ratio $p$-values. The dashed black curve corresponds to the combined search without the corrections discussed in the text. Right: Best fitted exotic production cross section. The green and yellow bands represent the one and two sigma variations around the median values. 

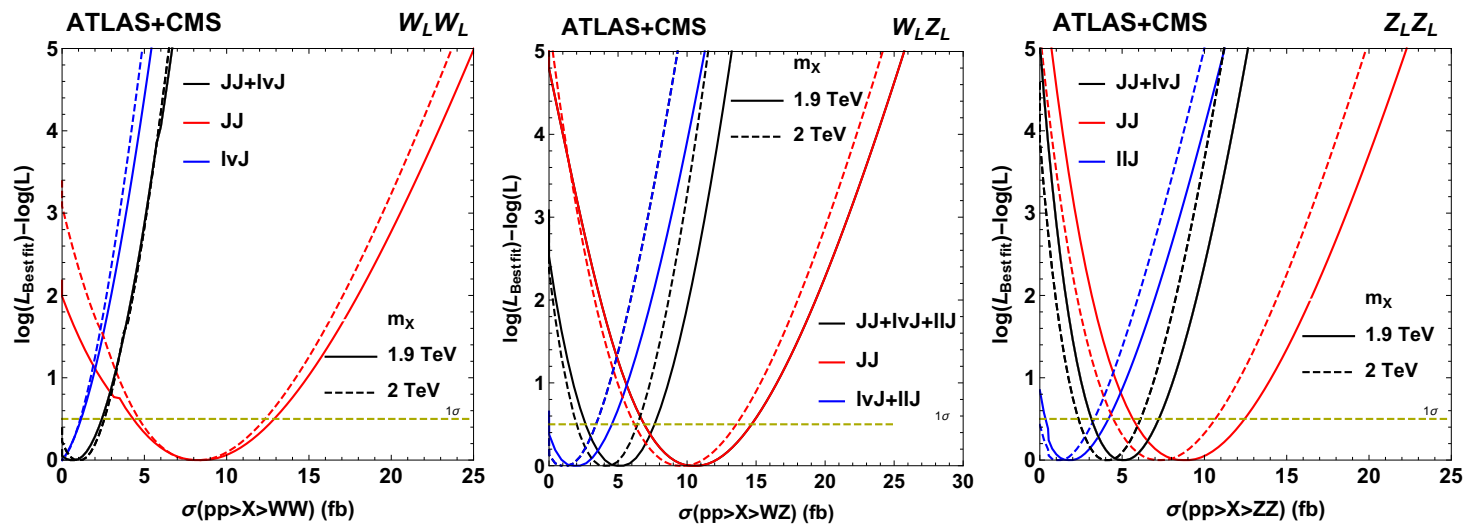

Figure 22. Combination of all ATLAS and CMS resonance searches: scans of the profile likelihood as a function of the production cross section for a $m_{\mathrm{X}}=2.0$ (1.9) $\mathrm{TeV}$ signal shown with continuous (dashed) lines in the hadronic (red) and semileptonic (blue) channels and their combination (black) for $\mathrm{W}_{\mathrm{L}} \mathrm{W}_{\mathrm{L}}$ (left), $\mathrm{W}_{\mathrm{L}} \mathrm{Z}_{\mathrm{L}}$ (middle) and $\mathrm{Z}_{\mathrm{L}} \mathrm{Z}_{\mathrm{L}}$ (right) selections and signal hypotheses.
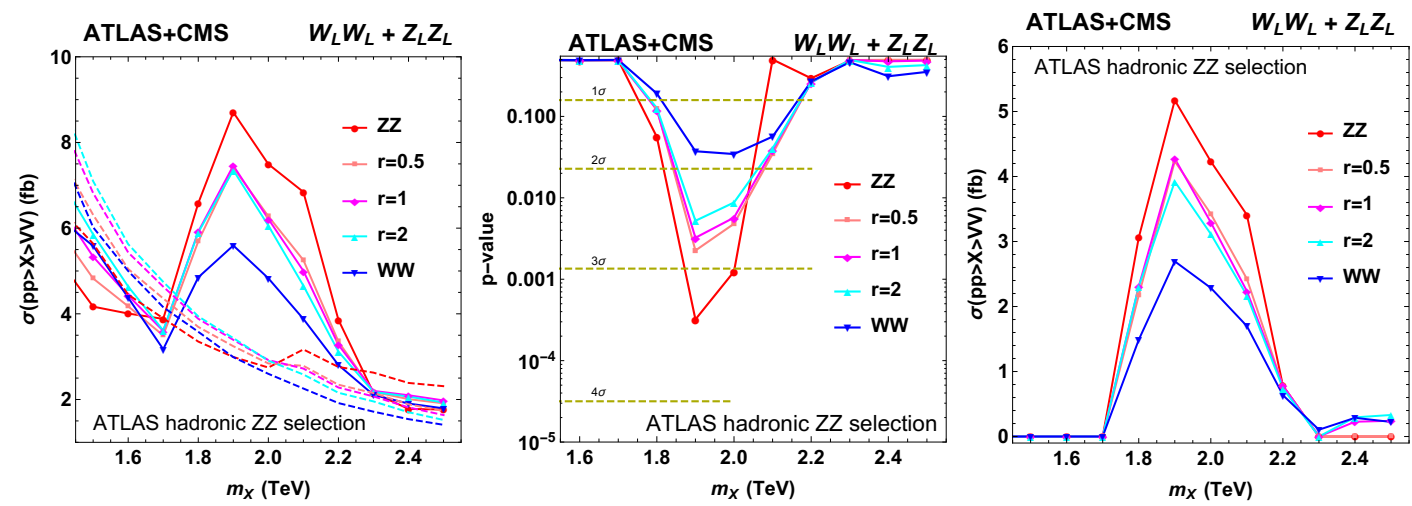

Figure 23. Combination of all ATLAS and CMS resonance searches, and dependence of results obtained in this study on the $r \equiv \mathcal{B}(X \rightarrow \mathrm{WW}) / \mathcal{B}(X \rightarrow \mathrm{ZZ})$ parameter for a neutral bulk RS-like spin-2 particle hypothesis, and as a function of the resonance mass $m_{\mathrm{X}}$. Left: expected (dashed lines) and observed (continuous lines) exclusion limits on exotic production cross section. Middle: likelihood-ratio $p$-values. Right: best fitted exotic production cross section.

\section{Conclusions}

We have performed a combination of the ATLAS and CMS searches for a heavy resonance decaying to a diboson final state, derived from the public information available for the six relevant analyses [20-22, 79, 80]. We have developed a methodology for the combination procedure, which begins with the work to emulate the public results by ATLAS and CMS for each individual analysis. This process is adjusted when necessary with correction factors to account for unknown uncertainties, and has been validated by reproducing the official results by the two experiments. We have presented combinations of the ATLAS and CMS searches for individual decay modes in various simplified models. At each step, the 95\% CL limits, the likelihood ratio $p$-values, the profile likelihood scans, and the maximum likelihood fits of the production cross section as function of the resonance mass $m_{\mathrm{X}}$ are provided. 


\begin{tabular}{|cccccc|}
\hline Signal hypothesis & $m_{\mathrm{X}}(\mathrm{TeV})$ & Significance & $p$-value & \multicolumn{2}{c|}{ Best-fit cross section $(\mathrm{fb})$} \\
\hline \multirow{2}{*}{$\mathrm{W}^{\prime} \rightarrow \mathrm{W}_{L} \mathrm{Z}_{L}$} & 1.9 & $2.5(3.1)$ & $6.5(1.0) \times 10^{-3}$ & $5.3_{-2.0}^{+2.3}$ & $\left(5.5_{-1.6}^{+2.0}\right)$ \\
& 2.0 & $2.5(3.2)$ & $7.0(0.8) \times 10^{-3}$ & $4.3_{-1.5}^{+2.1}$ & $\left(4.7_{-1.3}^{+1.8}\right)$ \\
$\mathrm{G}_{\text {bulk }} \rightarrow \mathrm{W}_{L} \mathrm{~W}_{L}$ & 1.9 & $0.49(0.83)$ & $0.30(0.20)$ & $0.75_{-0.75}^{+1.67}$ & $\left(1.4_{-1.4}^{+1.7}\right)$ \\
& 2.0 & $0.88(1.33)$ & $0.20(0.092)$ & $1.1_{-1.1}^{+1.4}$ & $\left(1.8_{-1.4}^{+1.8}\right)$ \\
$\mathrm{G}_{\text {bulk }} \rightarrow \mathrm{Z}_{L} \mathrm{Z}_{L}$ & 1.9 & $3.4(3.8)$ & $3.2(0.65) \times 10^{-4}$ & $5.2_{-1.6}^{+2.1}$ & $\left(4.7_{-1.2}^{+1.8}\right)$ \\
& 2.0 & $3.0(3.5)$ & $1.2(0.24) \times 10^{-3}$ & $4.2_{-1.2}^{+1.9}$ & $\left(3.9_{-1.0}^{+1.6}\right)$ \\
\hline \multirow{2}{*}{$\mathrm{G}_{\text {bulk }}(\mathrm{r}=2)$} & 1.9 & $2.6(3.4)$ & $5.2(0.40) \times 10^{-3}$ & $3.9_{-1.5}^{+2.4}$ & $\left(4.9_{-1.7}^{+2.0}\right)$ \\
& 2.0 & $2.4(3.1)$ & $8.8(0.89) \times 10^{-3}$ & $3.1_{-1.3}^{+1.8}$ & $\left(3.9_{-1.4}^{+1.6}\right)$ \\
\hline
\end{tabular}

Table 3. Summary of results obtained in this study: significance, $p$-values and best-fit cross sections for different model interpretations at $m_{\mathrm{X}}=1.9$ and $m_{\mathrm{X}}=2.0 \mathrm{TeV}$, i.e. the mass values where the largest excesses have been observed for different models. Our main results contain corrections that have been introduced to account for unknown uncertainties in the official results. (Additional results calculated without these correction factors are given inside the parentheses.)

The combination is obtained in three scenarios: $\mathrm{W}^{\prime} \rightarrow \mathrm{W}_{L} \mathrm{Z}_{L}, \mathrm{G}_{\text {bulk }} \rightarrow \mathrm{W}_{L} \mathrm{~W}_{L}$, and $\mathrm{G}_{\text {bulk }} \rightarrow \mathrm{Z}_{L} \mathrm{Z}_{L}$. We also obtain the full combination results for a $\mathrm{G}_{\text {bulk }}$ resonance with generic $\mathrm{W}_{\mathrm{L}} \mathrm{W}_{\mathrm{L}}$ and $\mathrm{Z}_{\mathrm{L}} \mathrm{Z}_{\mathrm{L}}$ branching fractions. Out of all benchmark models considered, the combination favours the hypothesis of a resonance with mass $1.9-2.0 \mathrm{TeV}$ and a production cross section $\approx 5 \mathrm{fb}$, as long as the resonance does not decay exclusively to $\mathrm{W}_{\mathrm{L}} \mathrm{W}_{\mathrm{L}}$ final states. Depending on the details of the resonance model, a signal significance between 2.4 and $3.4 \sigma$ is obtained for notable benchmark scenarios (see table 3). In particular, the possibility of a $\mathrm{W}^{\prime}$ resonance, suggested by other searches in different final states, is corroborated by the diboson searches, with a significance of $\approx 3 \sigma$ for a resonance mass of $1.9 \mathrm{TeV}$.

Note added. While preparing this manuscript for submission, ATLAS and CMS presented preliminary results in searches for diboson resonances with the first $\sqrt{s}=13 \mathrm{TeV}$ $p p$ collision data. They include results in the $W(\ell \nu) V(q \bar{q})$ [83], $Z\left(\ell^{+} \ell^{-}\right) V(q \bar{q})$ [84], $V(q \bar{q}) V(q \bar{q})[85]$ and $Z(\nu \bar{\nu}) V(q \bar{q})[86]$ channels by ATLAS, and the $W(\ell \nu) V(q \bar{q})$ and $V(q \bar{q}) V(q \bar{q})$ channels by CMS [87]. No significant excess above the SM expectations is observed, however the experimental sensitivity is, in most cases, not comparable with the one from Run-1 yet. The notable exception is the newly added $Z(\nu \bar{\nu}) V(q \bar{q})$ channel. The most stringent exclusion limits in the preliminary analysis of Run-2 data are obtained in the following channels:

- $(\mathrm{HVT}) \mathrm{W}^{\prime} \rightarrow \mathrm{W}_{L} \mathrm{Z}_{L}: 25 \mathrm{fb}(20 \mathrm{fb})$ for $m_{X}=1.9 \mathrm{TeV}(2.0 \mathrm{TeV})$ in the $W(q \bar{q}) Z(\nu \bar{\nu})$ channel by ATLAS, and the combination of the two channels considered by CMS.

- $\mathrm{G}_{\text {bulk }} \rightarrow \mathrm{W}_{L} \mathrm{~W}_{L}: 15 \mathrm{fb}(12 \mathrm{fb})$ for $m_{X}=1.9 \mathrm{TeV}(2.0 \mathrm{TeV})$ in the $W(\ell \nu) W(q \bar{q})$ channel by ATLAS. 
- $\mathrm{G}_{\mathrm{bulk}} \rightarrow \mathrm{Z}_{L} \mathrm{Z}_{L}: 21 \mathrm{fb}(15 \mathrm{fb})$ for $m_{X}=1.9 \mathrm{TeV}(2.0 \mathrm{TeV})$ in the $Z(\nu \bar{\nu}) Z(q \bar{q})$ channel by ATLAS.

In assessing the compatibility of the Run- 2 exclusion limits with the results obtained in this study (summarised in table 3) we use parton luminosity ratio values of 13 (15) for $m_{X}=1.9 \mathrm{TeV}(2.0 \mathrm{TeV})$ for $g g$ production $\left(\mathrm{G}_{\mathrm{bulk}} \rightarrow \mathrm{W}_{L} \mathrm{~W}_{L}\right.$ and $\mathrm{G}_{\mathrm{bulk}} \rightarrow \mathrm{Z}_{L} \mathrm{Z}_{L}$ channels $)$ and 8 (8.5) for $m_{X}=1.9 \mathrm{TeV}(2.0 \mathrm{TeV})$ for $q \bar{q}$ production $\left(\mathrm{W}^{\prime} \rightarrow \mathrm{W}_{L} \mathrm{Z}_{L}\right.$ channels) [88] to calculate the increase in the exotic signal production cross section from 8 to $13 \mathrm{TeV}$. We observe that the absence of a significant deviation in the Run-2 data

- creates a $\sim 2-3 \sigma$ tension with the best-fit cross section derived in this paper in the $\mathrm{G}_{\text {bulk }} \rightarrow \mathrm{Z}_{L} \mathrm{Z}_{L}$ channel,

- is consistent (within $1 \sigma$ ) with the (consistent-with-zero) result we obtain in the $\mathrm{G}_{\text {bulk }} \rightarrow \mathrm{W}_{L} \mathrm{~W}_{L}$ channel, and

- is also consistent (within $1 \sigma$ ) with the best-fit cross section that we have derived in the $\mathrm{W}^{\prime} \rightarrow \mathrm{W}_{L} \mathrm{Z}_{L}$ channel.

We, therefore, conclude that the preliminary analysis of the Run-2 data by ATLAS and CMS does not rule out the small deviation reported in the $\mathrm{W}^{\prime} \rightarrow \mathrm{W}_{L} \mathrm{Z}_{L}$ channel of the Run-1 diboson searches. It is widely expected that a clear picture will emerge with the analysis of the larger $13 \mathrm{TeV}$ datasets.

\section{Acknowledgments}

We would like to thank our colleagues at the ATLAS and CMS collaborations for their exemplary work and publication of a large number of papers on exotic searches. We thank Andreas Hinzmann for his precious help in the implementation of the CMS search in the $X \rightarrow \mathrm{VV} \rightarrow$ JJ channel. We also thank Goran Senjanović and Andrea Wulzer for fruitful discussions and valuable suggestions. A.O. thanks the CERN theory group for their hospitality.

This material is based upon work partially supported by the Cooperation Agreement (SPRINT Program) between the São Paulo Research Foundation (FAPESP) and the University of Edinburgh, under Grant No. 2014/50208-0. A.O. is supported by the MIURFIRB RBFR12H1MW grant. The work of F. D. and C.L. is supported by the Science and Technology Facilities Council (STFC) in the UK.

\section{A Comparison of different approaches to emulate ATLAS VV $\rightarrow$ JJ analysis}

The expected limits obtained in the emulation of the ATLAS VV $\rightarrow$ JJ channel show a $40 \%$ discrepancy with respect to the official results (see section 3). This is the largest discrepancy observed among all the channels considered in this study. We have considered alternative approaches in our strategy and carried out several cross-checks, which are summarised here: 

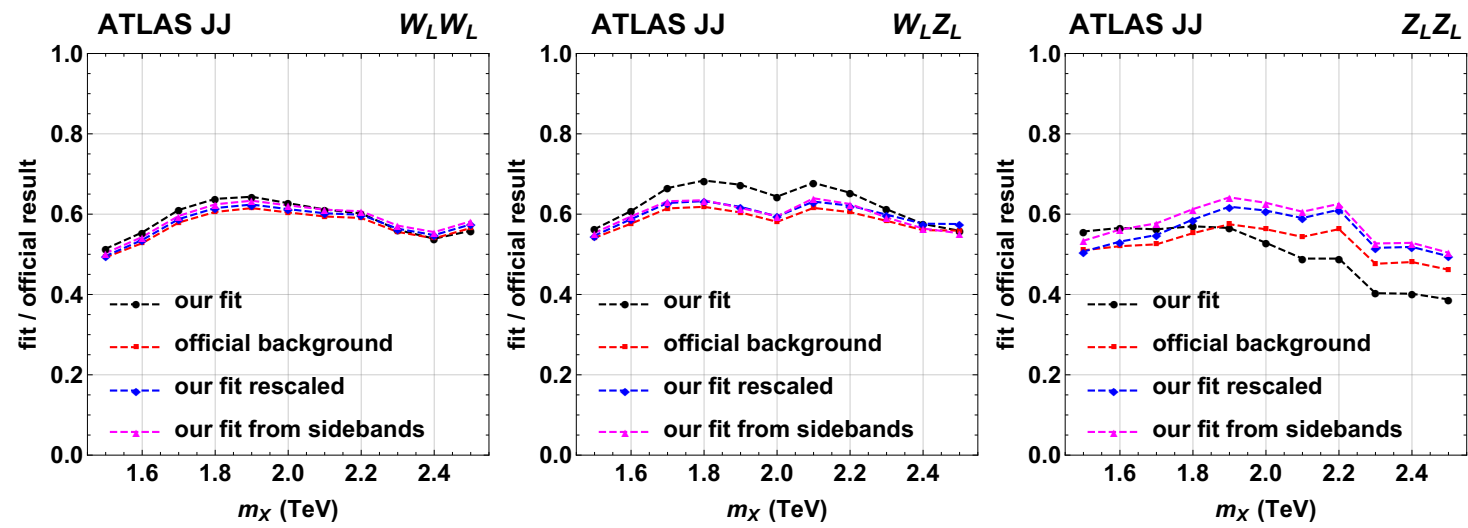

Figure 24. Emulation of ATLAS VV $\rightarrow$ JJ search and comparison of the alternative approaches for the background prediction considered: fudge factors as a function of the resonance mass $m_{\mathrm{X}}$, determined via the ratio of the expected limits obtained with different background estimation techniques (black: "pure fitting", red: "nominal background", blue: "rescaling", magenta: "sidebands") over those in the official ATLAS result for the $\mathrm{W}_{\mathrm{L}} \mathrm{W}_{\mathrm{L}}$ (left), $\mathrm{W}_{\mathrm{L}} \mathrm{Z}_{\mathrm{L}}$ (middle) and $\mathrm{Z}_{\mathrm{L}} \mathrm{Z}_{\mathrm{L}}$ channels (right). See text for details.

- Nominal background: ATLAS publishes a background description with a total background uncertainty. This information can be used directly as an input to our analysis. The disadvantage of this approach is that it combines all systematic uncertainties into a single contribution, implying a correlation model that may not reflect the accuracy of the fit performed by the ATLAS collaboration.

- Pure fitting: we have repeated the fit on the data distribution provided by the ATLAS collaboration. The fitting procedure naturally yields a covariance matrix for the shape parameters, which allows to adopt a more realistic correlation model.

- Rescaling: this is a mixed approach in which the fit is performed over the data distribution to obtain the covariance matrix of the fitting function parameters, but the resulting background prediction and the corresponding uncertainties are then rescaled to match those provided by ATLAS. In this approach, the official ATLAS background prediction is used and our fit is only used to model the uncertainties and their correlations.

- Sidebands: in this case we repeat the fit procedure described above, after excluding the region of the largest deviation $(1700-2300 \mathrm{GeV})$ from the fit range, in order to exclude the possibility that it could bias the fit.

Figure 24 shows the ratio of the observed exclusion limits to the ones from the official ATLAS results for the different approaches summarised above. In all cases the differences are very small, which suggests that the explanation for the observed discrepancy should be attributed to a factor other than the background determination procedure. The discrepancy is absorbed in the fudge factor which, when tuned to deliver the official expected exclusion limits, remarkably removes (to a large extent) the differences in the observed limits. One 

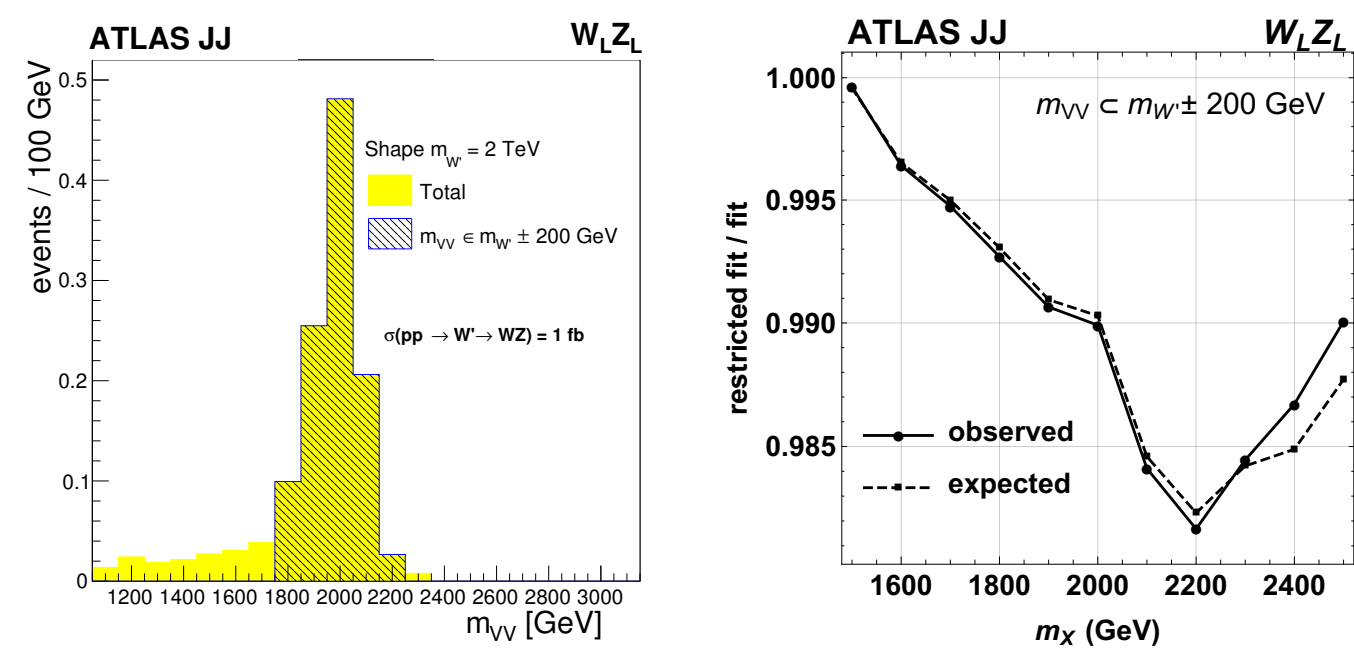

Figure 25. Narrow-width approximation. Left: signal distribution in the diboson invariant mass for a $2 \mathrm{TeV} \mathrm{W}^{\prime}$ signal. The hatched $\pm 200 \mathrm{GeV}$ region around the signal represents the narrow-width approximation. Right: ratio of the expected (dashed lines) and observed (continuous lines) exclusion limits when constraining the signal width to $10 \%$ of the resonance mass over those obtained with the default shape.

should note that the decision to employ these correction factors in our analysis (for this and other channels) does not change qualitatively the conclusions of this study. This can be seen, for example, in the middle plot of figure 21, where it is shown that the two different approaches yield significances that differer typically by $0.5 \sigma$.

\section{B Narrow width approximation}

The CMS collaboration assumes a signal with negligible width, whereas the ATLAS collaboration simulates signal distributions with a model-dependent width of $\approx 7 \%$ of the resonance mass (see table 1 of ref. [20]). In this appendix we estimate the effect of this difference in the final exclusion limits and provide a recipe for obtaining the ATLAS results in the narrow-width approximation.

The large width hypothesis used by the ATLAS collaboration impacts the limits through the modification of the signal shapes. In the JJ channel it widens the core for the signal distribution and creates a large left tail due to the interplay between proton PDFs [89] and the natural width of the resonance, as one can see in the left plot of figure 25. In practice, for a given total cross section we have events leaking outside the $\pm 10 \%$ window around $m_{\mathrm{X}}$. This value corresponds typically to the experimental resolution of this channel. The amount of this leakage, $f_{l}$ is provided in ref. [20] and corresponds typically to $15 \%$ in the region under study in this paper.

We expect the events in the left tail to have no significant impact on the exclusion limits. A test was performed by truncating the signal to $m_{\mathrm{X}} \pm 200 \mathrm{GeV}$ and repeating the JJ limit-setting procedure for the $\mathrm{W}^{\prime}$ hypothesis. As one can see in the right plot of figure 25 , the difference in the expected exclusion limits does not exceed $2 \%$. To map the 
ATLAS limits into a narrow width hypothesis we make the following approximation: the main difference between the wide and narrow resonances is the presence of leaking events in the right tail or under the peak. Consequently, by multiplying the signal efficiency of ATLAS by $1 / f_{l}$ we recover most of the properties of the narrow signal. In conclusion, we approximate the narrow signal hypothesis for ATLAS analyses by scaling the fully hadronic and semi-leptonic signals by a factor of 1.1 (i.e. by increasing the signal yield by $10 \%$ ).

Open Access. This article is distributed under the terms of the Creative Commons Attribution License (CC-BY 4.0), which permits any use, distribution and reproduction in any medium, provided the original author(s) and source are credited.

\section{References}

[1] J.C. Pati and A. Salam, Lepton Number as the Fourth Color, Phys. Rev. D 10 (1974) 275 [Erratum ibid. D 11 (1975) 703] [INSPIRE].

[2] R.N. Mohapatra and J.C. Pati, A Natural Left-Right Symmetry, Phys. Rev. D 11 (1975) 2558 [INSPIRE].

[3] G. Senjanović and R.N. Mohapatra, Exact Left-Right Symmetry and Spontaneous Violation of Parity, Phys. Rev. D 12 (1975) 1502 [inSPIRE].

[4] M.J. Dugan, H. Georgi and D.B. Kaplan, Anatomy of a Composite Higgs Model, Nucl. Phys. B 254 (1985) 299 [INSPIRE].

[5] H. Georgi and D.B. Kaplan, Composite Higgs and Custodial SU(2), Phys. Lett. B 145 (1984) 216 [INSPIRE].

[6] L. Randall and R. Sundrum, An Alternative to compactification, Phys. Rev. Lett. 83 (1999) 4690 [hep-th/9906064] [INSPIRE].

[7] L. Randall and R. Sundrum, A Large mass hierarchy from a small extra dimension, Phys. Rev. Lett. 83 (1999) 3370 [hep-ph/9905221] [INSPIRE].

[8] H. Davoudiasl, J.L. Hewett and T.G. Rizzo, Experimental probes of localized gravity: On and off the wall, Phys. Rev. D 63 (2001) 075004 [hep-ph/0006041] [inSPIRE].

[9] Tevatron Electroweak Working Group, CDF, DelPhi, Sld Electroweak and Heavy Flavour Groups, AlePh, LeP Electroweak Working Group, SLD, OPAL, D0, L3 and L.E.W. GRoup collaborations, Precision Electroweak Measurements and Constraints on the Standard Model, arXiv:1012.2367 [INSPIRE].

[10] M. Ciuchini, E. Franco, S. Mishima and L. Silvestrini, Electroweak Precision Observables, New Physics and the Nature of a 126 GeV Higgs Boson, JHEP 08 (2013) 106 [arXiv: 1306.4644] [INSPIRE].

[11] GFitTer Group collaboration, M. Baak et al., The global electroweak fit at NNLO and prospects for the LHC and ILC, Eur. Phys. J. C 74 (2014) 3046 [arXiv:1407.3792] [INSPIRE].

[12] J.M. Butterworth, A.R. Davison, M. Rubin and G.P. Salam, Jet substructure as a new Higgs search channel at the LHC, Phys. Rev. Lett. 100 (2008) 242001 [arXiv:0802.2470] [INSPIRE]. 
[13] S.D. Ellis, C.K. Vermilion and J.R. Walsh, Recombination Algorithms and Jet Substructure: Pruning as a Tool for Heavy Particle Searches, Phys. Rev. D 81 (2010) 094023 [arXiv: 0912.0033] [INSPIRE].

[14] I.W. Stewart, F.J. Tackmann and W.J. Waalewijn, N-Jettiness: An Inclusive Event Shape to Veto Jets, Phys. Rev. Lett. 105 (2010) 092002 [arXiv: 1004.2489] [InSPIRE].

[15] J. Thaler and K. Van Tilburg, Identifying Boosted Objects with $N$-subjettiness, JHEP 03 (2011) 015 [arXiv: 1011.2268] [INSPIRE].

[16] J. Thaler and K. Van Tilburg, Maximizing Boosted Top Identification by Minimizing $N$-subjettiness, JHEP 02 (2012) 093 [arXiv: 1108.2701] [INSPIRE].

[17] M. Gouzevitch, A. Oliveira, J. Rojo, R. Rosenfeld, G.P. Salam and V. Sanz, Scale-invariant resonance tagging in multijet events and new physics in Higgs pair production, JHEP $\mathbf{0 7}$ (2013) 148 [arXiv:1303.6636] [InSPIRE].

[18] CMS collaboration, Identification techniques for highly boosted $W$ bosons that decay into hadrons, JHEP 12 (2014) 017 [arXiv:1410.4227] [INSPIRE].

[19] ATLAS collaboration, Identification of boosted, hadronically decaying $W$ bosons and comparisons with ATLAS data taken at $\sqrt{s}=8$ TeV, Eur. Phys. J. C 76 (2016) 154 [arXiv: 1510.05821] [INSPIRE].

[20] ATLAS collaboration, Search for high-mass diboson resonances with boson-tagged jets in proton-proton collisions at $\sqrt{s}=8 \mathrm{TeV}$ with the ATLAS detector, JHEP 12 (2015) 055 [arXiv: 1506.00962] [INSPIRE].

[21] CMS collaboration, Search for massive resonances decaying into pairs of boosted bosons in semi-leptonic final states at $\sqrt{s}=8 \mathrm{TeV}$, JHEP 08 (2014) 174 [arXiv:1405.3447] [INSPIRE].

[22] CMS collaboration, Search for massive resonances in dijet systems containing jets tagged as $W$ or $Z$ boson decays in pp collisions at $\sqrt{s}=8 \mathrm{TeV}$, JHEP 08 (2014) 173 [arXiv: 1405.1994] [INSPIRE].

[23] ATLAS collaboration, Search for new phenomena in the dijet mass distribution using $p-p$ collision data at $\sqrt{s}=8 \mathrm{TeV}$ with the ATLAS detector, Phys. Rev. D 91 (2015) 052007 [arXiv: 1407.1376] [INSPIRE].

[24] ATLAS collaboration, Search for new phenomena in dijet mass and angular distributions from pp collisions at $\sqrt{s}=13 \mathrm{TeV}$ with the ATLAS detector, Phys. Lett. B 754 (2016) 302 [arXiv: 1512.01530] [INSPIRE].

[25] CMS collaboration, Search for resonances and quantum black holes using dijet mass spectra in proton-proton collisions at $\sqrt{s}=8 \mathrm{TeV}$, Phys. Rev. D 91 (2015) 052009 [arXiv: 1501.04198] [INSPIRE].

[26] CMS collaboration, Search for narrow resonances decaying to dijets in proton-proton collisions at $\sqrt{s}=13 \mathrm{TeV}$, Phys. Rev. Lett. 116 (2016) 071801 [arXiv:1512.01224] [INSPIRE].

[27] CMS collaboration, Search for physics beyond the standard model in dilepton mass spectra in proton-proton collisions at $\sqrt{s}=8 \mathrm{TeV}$, JHEP 04 (2015) 025 [arXiv:1412.6302] [INSPIRE].

[28] W.-Y. Keung and G. Senjanović, Majorana Neutrinos and the Production of the Right-handed Charged Gauge Boson, Phys. Rev. Lett. 50 (1983) 1427 [InSPIRE]. 
[29] CMS collaboration, Search for heavy neutrinos and $\mathrm{W}$ bosons with right-handed couplings in proton-proton collisions at $\sqrt{s}=8 \mathrm{TeV}$, Eur. Phys. J. C 74 (2014) 3149 [arXiv:1407.3683] [INSPIRE].

[30] ATLAS collaboration, Search for heavy Majorana neutrinos with the ATLAS detector in $p p$ collisions at $\sqrt{s}=8 \mathrm{TeV}$, JHEP 07 (2015) 162 [arXiv:1506.06020] [INSPIRE].

[31] CMS collaboration, Search for massive $W H$ resonances decaying into the $\ell \nu \mathrm{b} \overline{\mathrm{b}}$ final state at $\sqrt{s}=8 \mathrm{TeV}$, arXiv:1601.06431 [INSPIRE].

[32] CMS collaboration, Search for a massive resonance decaying into a Higgs boson and a $W$ or $Z$ boson in hadronic final states in proton-proton collisions at $\sqrt{s}=8 \mathrm{TeV}$, JHEP 02 (2016) 145 [arXiv: 1506.01443] [INSPIRE].

[33] CMS collaboration, Search for Narrow High-Mass Resonances in Proton-Proton Collisions at $\sqrt{s}=8$ TeV Decaying to a $Z$ and a Higgs Boson, Phys. Lett. B 748 (2015) 255 [arXiv: 1502.04994] [INSPIRE].

[34] CMS collaboration, Search for resonant pair production of Higgs bosons decaying to $b \bar{b}$ and $\tau^{+} \tau^{-}$in proton-proton collisions at $\sqrt{s}=8 \mathrm{TeV}$, CMS-PAS-EXO-15-008 (2015).

[35] J. Hisano, N. Nagata and Y. Omura, Interpretations of the ATLAS Diboson Resonances, Phys. Rev. D 92 (2015) 055001 [arXiv:1506.03931] [INSPIRE].

[36] K. Cheung, W.-Y. Keung, P.-Y. Tseng and T.-C. Yuan, Interpretations of the ATLAS Diboson Anomaly, Phys. Lett. B 751 (2015) 188 [arXiv:1506.06064] [INSPIRE].

[37] B.A. Dobrescu and Z. Liu, W'Boson near 2 TeV: Predictions for Run 2 of the LHC, Phys. Rev. Lett. 115 (2015) 211802 [arXiv:1506.06736] [INSPIRE].

[38] Y. Gao, T. Ghosh, K. Sinha and J.-H. Yu, SU(2) $\times \mathrm{SU}(2) \times \mathrm{U}(1)$ interpretations of the diboson and Wh excesses, Phys. Rev. D 92 (2015) 055030 [arXiv: 1506.07511] [INSPIRE].

[39] J. Brehmer, J. Hewett, J. Kopp, T. Rizzo and J. Tattersall, Symmetry Restored in Dibosons at the LHC?, JHEP 10 (2015) 182 [arXiv: 1507.00013] [INSPIRE].

[40] T. Abe, R. Nagai, S. Okawa and M. Tanabashi, Unitarity sum rules, three-site moose model and the ATLAS $2 \mathrm{TeV}$ diboson anomalies, Phys. Rev. D 92 (2015) 055016 [arXiv: 1507.01185] [INSPIRE].

[41] P.S. Bhupal Dev and R.N. Mohapatra, Unified explanation of the eejj, diboson and dijet resonances at the LHC, Phys. Rev. Lett. 115 (2015) 181803 [arXiv:1508.02277] [INSPIRE].

[42] P. Coloma, B.A. Dobrescu and J. Lopez-Pavon, Right-handed neutrinos and the $2 \mathrm{TeV} W^{\prime}$ boson, Phys. Rev. D 92 (2015) 115023 [arXiv: 1508.04129] [INSPIRE].

[43] Q.-H. Cao, B. Yan and D.-M. Zhang, Simple non-Abelian extensions of the standard model gauge group and the diboson excesses at the LHC, Phys. Rev. D 92 (2015) 095025 [arXiv: 1507.00268] [INSPIRE].

[44] K. Das, T. Li, S. Nandi and S.K. Rai, Diboson excesses in an anomaly free leptophobic left-right model, Phys. Rev. D 93 (2016) 016006 [arXiv:1512.00190] [INSPIRE].

[45] J.A. Aguilar-Saavedra, Triboson interpretations of the ATLAS diboson excess, JHEP 10 (2015) 099 [arXiv: 1506. 06739] [INSPIRE].

[46] G. Cacciapaglia and M.T. Frandsen, Unitarity implications of a diboson resonance in the TeV region for Higgs physics, Phys. Rev. D 92 (2015) 055035 [arXiv: 1507.00900] [INSPIRE]. 
[47] B.C. Allanach, B. Gripaios and D. Sutherland, Anatomy of the ATLAS diboson anomaly, Phys. Rev. D 92 (2015) 055003 [arXiv: 1507.01638] [InSPIRE].

[48] T. Abe, T. Kitahara and M.M. Nojiri, Prospects for Spin-1 Resonance Search at $13 \mathrm{TeV}$ LHC and the ATLAS Diboson Excess, JHEP 02 (2016) 084 [arXiv:1507.01681] [INSPIRE].

[49] H.S. Fukano, S. Matsuzaki and K. Yamawaki, Conformal Barrier for New Vector Bosons Decay to the Higgs, Mod. Phys. Lett. A 31 (2016) 1630009 [arXiv:1507.03428] [inSPIRE].

[50] S.P. Liew and S. Shirai, Testing ATLAS Diboson Excess with Dark Matter Searches at LHC, JHEP 11 (2015) 191 [arXiv:1507.08273] [INSPIRE].

[51] J.H. Collins and W.H. $\mathrm{Ng}, A 2 \mathrm{TeV} W_{R}$, supersymmetry and the Higgs mass, JHEP 01 (2016) 159 [arXiv : 1510.08083] [INSPIRE].

[52] D. Aristizabal Sierra, J. Herrero-Garcia, D. Restrepo and A. Vicente, Diboson anomaly: Heavy Higgs resonance and QCD vectorlike exotics, Phys. Rev. D 93 (2016) 015012 [arXiv: 1510.03437] [INSPIRE].

[53] H.S. Fukano, M. Kurachi, S. Matsuzaki, K. Terashi and K. Yamawaki, 2 TeV Walking Technirho at LHC?, Phys. Lett. B 750 (2015) 259 [arXiv: 1506.03751] [InSPIRE].

[54] D.B. Franzosi, M.T. Frandsen and F. Sannino, Diboson Signals via Fermi Scale Spin-One States, Phys. Rev. D 92 (2015) 115005 [arXiv:1506.04392] [InSPIRE].

[55] A. Thamm, R. Torre and A. Wulzer, Composite Heavy Vector Triplet in the ATLAS Diboson Excess, Phys. Rev. Lett. 115 (2015) 221802 [arXiv:1506.08688] [InSPIRE].

[56] C.-W. Chiang, H. Fukuda, K. Harigaya, M. Ibe and T.T. Yanagida, Diboson Resonance as a Portal to Hidden Strong Dynamics, JHEP 11 (2015) 015 [arXiv: 1507.02483] [INSPIRE].

[57] M. Low, A. Tesi and L.-T. Wang, Composite spin-1 resonances at the LHC, Phys. Rev. D 92 (2015) 085019 [arXiv: 1507.07557] [INSPIRE].

[58] H.S. Fukano, S. Matsuzaki, K. Terashi and K. Yamawaki, Conformal Barrier and Hidden Local Symmetry Constraints: Walking Technirhos in LHC Diboson Channels, Nucl. Phys. B 904 (2016) 400 [arXiv: 1510.08184] [INSPIRE].

[59] A. Carmona, A. Delgado, M. Quirós and J. Santiago, Diboson resonant production in non-custodial composite Higgs models, JHEP 09 (2015) 186 [arXiv: 1507.01914] [INSPIRE].

[60] B.A. Dobrescu and Z. Liu, Heavy Higgs bosons and the 2 TeV W' boson, JHEP 10 (2015) 118 [arXiv: 1507.01923] [INSPIRE].

[61] V. Sanz, On the Compatibility of the Diboson Excess with a gg-Initiated Composite Sector, Adv. High Energy Phys. 2016 (2016) 3279568 [arXiv: 1507.03553] [INSPIRE].

[62] C.-H. Chen and T. Nomura, 2 TeV Higgs boson and diboson excess at the LHC, Phys. Lett. B 749 (2015) 464 [arXiv: 1507.04431 ] [INSPIRE].

[63] Y. Omura, K. Tobe and K. Tsumura, Survey of Higgs interpretations of the diboson excesses, Phys. Rev. D 92 (2015) 055015 [arXiv: 1507.05028] [INSPIRE].

[64] C.-H. Chen and T. Nomura, Diboson excess in the Higgs singlet and vectorlike quark models, Phys. Rev. D 92 (2015) 115021 [arXiv:1509.02039] [INSPIRE].

[65] H.M. Lee, D. Kim, K. Kong and S.C. Park, Diboson Excesses Demystified in Effective Field Theory Approach, JHEP 11 (2015) 150 [arXiv:1507.06312] [INSPIRE]. 
[66] F.F. Deppisch et al., Reconciling the $2 \mathrm{TeV}$ excesses at the LHC in a linear seesaw left-right model, Phys. Rev. D 93 (2016) 013011 [arXiv:1508.05940] [INSPIRE].

[67] A. Alves, D.A. Camargo and A.G. Dias, Heavy Higgs Coupled to Vector-Like Quarks: Strong CP Problem, Diboson Excess and Search Prospects at the 14 TeV LHC, arXiv:1511.04449 [INSPIRE].

[68] J. Brehmer et al., The Diboson Excess: Experimental Situation and Classification of Explanations; A Les Houches Pre-Proceeding, arXiv:1512.04357 [INSPIRE].

[69] HEPDATA database, http://hepdata.cedar.ac.uk/.

[70] T. Müller, J. Ott and J. Wagner-Kuhr, The theta framework for template-based statistical modeling and inference, http://www.theta-framework.org/.

[71] G. Cowan, K. Cranmer, E. Gross and O. Vitells, Asymptotic formulae for likelihood-based tests of new physics, Eur. Phys. J. C 71 (2011) 1554 [Erratum ibid. C 73 (2013) 2501] [arXiv: 1007.1727] [INSPIRE].

[72] Presentation of search results: the $C L_{s}$ technique, J. Phys. G 28 (2002) 2693.

[73] T. Junk, Confidence level computation for combining searches with small statistics, Nucl. Instrum. Meth. A 434 (1999) 435 [hep-ex/9902006] [INSPIRE].

[74] J. Alwall et al., The automated computation of tree-level and next-to-leading order differential cross sections and their matching to parton shower simulations, JHEP 07 (2014) 079 [arXiv: 1405.0301] [INSPIRE].

[75] T. Sjöstrand, S. Mrenna and P.Z. Skands, A Brief Introduction to PYTHIA 8.1, Comput. Phys. Commun. 178 (2008) 852 [arXiv:0710.3820] [INSPIRE].

[76] A. Oliveira, Gravity particles from Warped Extra Dimensions, a review. Part I - KK Graviton, arXiv:1404.0102 [INSPIRE].

[77] D. Pappadopulo, A. Thamm, R. Torre and A. Wulzer, Heavy Vector Triplets: Bridging Theory and Data, JHEP 09 (2014) 060 [arXiv: 1402.4431] [INSPIRE].

[78] ATLAS collaboration, Combination of searches for $W W, W Z$ and $Z Z$ resonances in $p p$ collisions at $\sqrt{s}=8 \mathrm{TeV}$ with the ATLAS detector, Phys. Lett. B 755 (2016) 285 [arXiv: 1512.05099] [INSPIRE].

[79] ATLAS collaboration, Search for production of $W W / W Z$ resonances decaying to a lepton, neutrino and jets in pp collisions at $\sqrt{s}=8$ TeV with the ATLAS detector, Eur. Phys. J. C 75 (2015) 209 [Erratum ibid. C 75 (2015) 370] [arXiv: 1503.04677] [INSPIRE].

[80] ATLAS collaboration, Search for resonant diboson production in the $\ell \ell q \bar{q}$ final state in $p p$ collisions at $\sqrt{s}=8 \mathrm{TeV}$ with the ATLAS detector, Eur. Phys. J. C 75 (2015) 69 [arXiv: 1409.6190] [INSPIRE].

[81] Y.L. Dokshitzer, G.D. Leder, S. Moretti and B.R. Webber, Better jet clustering algorithms, JHEP 08 (1997) 001 [hep-ph/9707323] [INSPIRE].

[82] M. Wobisch and T. Wengler, Hadronization corrections to jet cross-sections in deep inelastic scattering, in Proceedings of Monte Carlo generators for HERA physics Workshop, Hamburg Germany (1998) [hep-ph/9907280] [INSPIRE].

[83] ATLAS collaboration, Search for $W W / W Z$ resonance production in the $\ell \nu q q$ final state at $\sqrt{s}=13 \mathrm{TeV}$ with the ATLAS detector at the LHC, ATLAS-CONF-2015-075 (2015). 
[84] ATLAS collaboration, Search for diboson resonances in the llqq final state in pp collisions at $\sqrt{s}=13$ TeV with the ATLAS detector, ATLAS-CONF-2015-071 (2015).

[85] ATLAS collaboration, Search for resonances with boson-tagged jets in $3.2 \mathrm{fb}^{-1}$ of pp collisions at $\sqrt{s}=13$ TeV collected with the ATLAS detector, ATLAS-CONF-2015-073 (2015).

[86] ATLAS collaboration, Search for diboson resonances in the $\nu \nu q q$ final state in pp collisions at $\sqrt{s}=13 \mathrm{TeV}$ with the ATLAS detector, ATLAS-CONF-2015-068 (2015).

[87] CMS collaboration, Search for massive resonances decaying into pairs of boosted $W$ and $Z$ bosons at $\sqrt{s}=13 \mathrm{TeV}$, CMS-PAS-EXO-15-002 (2015).

[88] W.J. Stirling, private communication, http://www.hep.ph.ic.ac.uk/ wstirlin/plots/plots.html.

[89] R.M. Harris and K. Kousouris, Searches for Dijet Resonances at Hadron Colliders, Int. J. Mod. Phys. A 26 (2011) 5005 [arXiv:1110.5302] [INSPIRE]. 